\title{
HOMOMORPHISMS OF MULTIPLICATIVE GROUPS OF FIELDS PRESERVING ALGEBRAIC DEPENDENCE
}

\author{
FEDOR BOGOMOLOV, MARAT ROVINSKY, AND YURI TSCHINKEL

\begin{abstract}
We study homomorphisms of multiplicative groups of fields preserving algebraic dependence and show that such homomorphisms give rise to valuations.
\end{abstract}

\section{INTRODUCTION}

In this paper we formulate and prove a version of the Grothendieck section conjecture. For function fields of algebraic varieties over algebraically closed ground fields this conjecture states, roughly, that existence of group-theoretic sections of homomorphisms of their absolute Galois groups implies existence of geometric sections of morphisms of models of these fields.

In detail, let $k$ be an algebraically closed field and $K=k(X)$ the function field of an algebraic variety $X$ over $k$. Let $G_{K}$ be the absolute Galois group of $K$. Fix a prime $\ell$ not equal to the characteristic of $k$ and let $\mathcal{G}_{K}$ be the maximal pro- $\ell$-quotient of $G_{K}$, the Galois group of the maximal $\ell$-extension of $K$. Write

$$
\mathcal{G}_{K}^{a}=\mathcal{G}_{K} /\left[\mathcal{G}_{K}, \mathcal{G}_{K}\right] \quad \text { and } \quad \mathcal{G}_{K}^{c}:=\mathcal{G}_{K} /\left[\mathcal{G}_{K},\left[\mathcal{G}_{K}, \mathcal{G}_{K}\right]\right] \text {, }
$$

for the abelianization and its canonical central extension:

$$
1 \rightarrow \mathcal{Z}_{K} \rightarrow \mathcal{G}_{K}^{c} \stackrel{\pi_{a}}{\longrightarrow} \mathcal{G}_{K}^{a} \rightarrow 1 .
$$

Let $\Sigma_{K}=\Sigma\left(\mathcal{G}_{K}^{c}\right)$ be the set of topologically noncyclic subgroups $\sigma \subset$ $\mathcal{G}_{K}^{a}$ whose preimages $\pi_{a}^{-1}(\sigma) \subset \mathcal{G}_{K}^{c}$ are abelian. It is known that function fields $K=k(X)$ of transcendence degree $\geq 2$ over $k=\overline{\mathbb{F}}_{p}$ are determined, modulo purely inseparable extensions, by the pair $\left(\mathcal{G}_{K}^{a}, \Sigma_{K}\right)$ [3], 4], and [7].

This raises the question of functoriality, i.e., the reconstruction of rational morphisms between algebraic varieties from continuous homomorphisms of absolute Galois groups of their function fields. This general fundamental question was proposed by Grothendieck and lies at the core of the Anabelian Geometry Program.

Key words and phrases. Field theory, valuations. 
The main open problem in this program relates to a Galois-theoretic criterium for the existence of rational sections of fibrations. Let

$$
\pi: X \rightarrow Y
$$

be a fibration with connected generic fiber of dimension at least 1 over a base $Y$ of dimension $\geq 2$. This defines a field embedding

$$
\pi^{*}: k(Y) \hookrightarrow k(X),
$$

with image of $L:=k(Y)$ algebraically closed in $K:=k(X)$. Dually, we have a surjective homomorphism of absolute Galois groups (restriction map)

$$
G_{K} \rightarrow G_{L}
$$

as well as induced homomorphisms

$$
\mathcal{G}_{K}^{c} \rightarrow \mathcal{G}_{L}^{c}, \quad \mathcal{G}_{K}^{a} \rightarrow \mathcal{G}_{L}^{a} .
$$

A minimalistic version of Grothendieck's Section conjecture would be:

Conjecture 1. Assume that $\pi_{a}: \mathcal{G}_{K}^{a} \rightarrow \mathcal{G}_{L}^{a}$ admits a section

$$
\xi_{a}: \mathcal{G}_{L}^{a} \rightarrow \mathcal{G}_{K}^{a}
$$

such that

$$
\xi_{a}\left(\Sigma_{L}\right) \subset \Sigma_{K}
$$

Then there exist a finite purely inseparable extension

$$
\iota^{*}: L \hookrightarrow L^{\prime}=k\left(Y^{\prime}\right)
$$

and a rational map

$$
\xi: Y^{\prime} \rightarrow X
$$

such that

$$
\xi^{*} \circ \pi^{*}(L)=\iota^{*}(L) \subset L^{\prime} .
$$

Thus $\xi\left(Y^{\prime}\right)$ is a section over $Y$, modulo purely inseparable extensions.

Conjecture 1 is closely related to questions considered in this note. Recall that, by Kummer theory,

$$
\mathcal{G}_{K}^{a}=\operatorname{Hom}\left(K^{\times}, \mathbb{Z}_{\ell}(1)\right)
$$

and that (2) induces the dual homomorphism of (profinite) pro- $\ell$-completions of multiplicative groups

$$
\hat{\psi}: \hat{K}^{\times} \rightarrow \hat{L}^{\times}
$$


Then (3) says that $\hat{\psi}$ respects the skew-symmetric pairings on $\hat{K}^{\times}$and $\hat{L}^{\times}$, with values in the second Galois cohomology group of the corresponding field. The groups $\hat{K}^{\times}$and $\hat{L}^{\times}$contain $K^{\times} / k^{\times}$and $L^{\times} / k^{\times}$, respectively. If the restriction $\psi$ of $\hat{\psi}$ to $K^{\times} / k^{\times}$is "rational", i.e.,

$$
\psi: K^{\times} / k^{\times} \subseteq L^{\times} / k^{\times} \subset \hat{L}^{\times},
$$

then $\psi$ respects algebraic dependence, mapping algebraically dependent elements in $K^{\times}$to algebraically dependent elements of $L^{\times}$(modulo $\left.k^{\times}\right)$. For function fields this is equivalent to (3). This relates the "minimalistic" version of the Section conjecture for "rational" maps to our main result, which we now explain.

Let $\nu$ be a nonarchimedean valuation of $K$, i.e., a homomorphism

$$
\nu: K^{\times} \rightarrow \Gamma_{\nu}
$$

onto a totally ordered group such that the induced map

$$
\nu: K \rightarrow \Gamma_{\nu} \cup\{\infty\}, \quad \nu(0)=\infty,
$$

satisfies a nonarchimedean triangle inequality. Let

$$
\mathfrak{m}_{K, \nu} \subset \mathfrak{o}_{K, \nu}, \quad \boldsymbol{K}_{\nu}:=\mathfrak{o}_{K, \nu} / \mathfrak{m}_{K, \nu}
$$

be the maximal ideal, valuation ring, and residue field with respect to $\nu$, respectively. If $K \mid k$ is a field extension and $\nu$ a valuation of $K$, then its restriction to $k$ is also a valuation; we have

$$
\mathfrak{o}_{K, \nu}^{\times} \cap k^{\times}=\mathfrak{o}_{k, \nu}^{\times}, \quad \mathfrak{o}_{K, \nu}^{\times} / \mathfrak{o}_{k, \nu}^{\times} \subseteq K^{\times} / k^{\times},
$$

and a natural surjection

$$
\mathfrak{o}_{K, \nu}^{\times} / \mathfrak{o}_{k, \nu}^{\times} \longrightarrow \boldsymbol{K}_{\nu}^{\times} / \boldsymbol{k}_{\nu}^{\times} .
$$

We consider extensions of fields

$$
k \subseteq \tilde{k} \subseteq \tilde{k}_{a} \subset K,
$$

where $k$ is the prime subfield of $K$, i.e., $k=\mathbb{F}_{p}$ or $\mathbb{Q}$, and $\tilde{k}_{a} \subset K$ the algebraic closure of $\tilde{k}$ in $K$, i.e., the set of all algebraic elements over $k$ contained in $K$. Assume that $\bar{x}_{1}, \bar{x}_{2} \in K^{\times} / k^{\times}$safisfy

$$
\operatorname{tr} \operatorname{deg}_{\tilde{k}}\left(\tilde{k}\left(x_{1}, x_{2}\right)\right) \leq 1,
$$

for their lifts $x_{1}, x_{2} \in K^{\times}$; this does not depend on the choice of lifts. We write $x_{1} \sim_{\tilde{k}} x_{2}$ and say that $x_{1}$ and $x_{2}$ are contained in the same one-dimensional field; clearly $1 \sim_{\tilde{k}} x$, for all $\bar{x} \in K^{\times} / k^{\times}$. From now on, we use the same notation for an element $x \in K^{\times}$and its image in $K^{\times} / k^{\times}$. Let

$$
l \subseteq \tilde{l} \subseteq \tilde{l}_{a} \subset L
$$


be field extensions, where $l$ is the prime subfield of $L$, and let

$$
\psi: K^{\times} / k^{\times} \rightarrow L^{\times} / \tilde{l}^{\times}
$$

be a homomorphism of multiplicative groups. We say that $\psi$ preserves algebraic dependence with respect to $\tilde{k}, \tilde{l}$ if

$$
x_{1} \sim_{\tilde{k}} x_{2} \Rightarrow \psi\left(x_{1}\right) \sim_{\tilde{l}} \psi\left(x_{2}\right), \quad \forall x_{1}, x_{2} \in K^{\times} / k^{\times} .
$$

Theorem 2. Let $k \subseteq \tilde{k} \subset K$ and $l \subseteq \tilde{l} \subset L$ be field extensions as above. Assume that $\tilde{l}=\tilde{l}_{a}$ and that there exists a homomorphism

$$
\psi: K^{\times} / k^{\times} \rightarrow L^{\times} / \tilde{l}^{\times},
$$

such that

- $\psi$ preserves algebraic dependence with respect to $\tilde{k}$ and $\tilde{l}$;

- there exist

$$
y_{1}, y_{2} \in \psi\left(K^{\times} / k^{\times}\right), \quad \text { such that } \quad y_{1} \chi_{\tilde{l}} y_{2} ;
$$

- $\psi$ satisfies Assumption (AD) of Section 7 .

Then either

(P) there exists a field $F \subset K$ such that $\psi$ factors through

$$
K^{\times} / k^{\times} \longrightarrow K^{\times} / F^{\times}
$$

$(\mathrm{V})$ there exists a nontrivial valuation $\nu$ on $K$ such that the restriction of $\psi$ to

$$
\mathfrak{o}_{K, \nu}^{\times} / \mathfrak{o}_{k, \nu}^{\times} \subseteq K^{\times} / k^{\times}
$$

is trivial on

$$
\left(1+\mathfrak{m}_{\nu}\right)^{\times} / \mathfrak{o}_{k, \nu}^{\times}
$$

and it factors through the reduction map

$$
\mathfrak{o}_{K, \nu}^{\times} / \mathfrak{o}_{k, \nu}^{\times} \longrightarrow \boldsymbol{K}_{\nu}^{\times} / \boldsymbol{k}_{\nu}^{\times} \rightarrow L^{\times} / \tilde{l}^{\times},
$$

(VP) there exist a nontrivial valuation $\nu$ on $K$ and a field $\boldsymbol{F}_{\nu} \subset \boldsymbol{K}_{\nu}$ such that the restriction of $\psi$ to $\mathfrak{o}_{K, \nu}^{\times} / \mathfrak{o}_{k, \nu}^{\times}$factors through

$$
\mathfrak{o}_{K, \nu}^{\times} / \mathfrak{o}_{k, \nu}^{\times} \longrightarrow \boldsymbol{K}_{\nu}^{\times} / \boldsymbol{F}_{\nu}^{\times} \rightarrow L^{\times} / \tilde{l}^{\times} .
$$

In the geometric setting treated in [5], when $K=\tilde{k}(X)$ is a function field of an algebraic variety $X$ over $\tilde{k}=\overline{\mathbb{F}}_{p}$, the center of the valuation $\nu$ arising in case $(\mathrm{V})$ is, birationally, the image of the section, and the above theorem can be viewed as a "rational" version of the minimalistic section conjecture. Here we extend the argument in [5] from function fields to arbitrary fields, under the additional technical assumption (AD) on $\psi$, which holds for $K$ of positive characteristic.

The idea of proof is to reduce the problem to a question in plane projective geometry over the prime subfield $k$. We view $\mathbb{P}(K):=K^{\times} / k^{\times}$ 
as a projective space over $k$. To establish Theorem 2 it suffices to show the existence of a subgroup $\mathfrak{U} \subset K^{\times} / k^{\times}$such that:

Condition 3. For every projective line $\mathfrak{l} \subset \mathbb{P}(K), \mathfrak{U} \cap \mathfrak{l}$ is either

(1) the line $\mathfrak{l}$,

(2) a point $\mathfrak{q} \in \mathfrak{l}$,

(3) the affine line $\mathfrak{l} \backslash \mathfrak{q}$, or

(4) if $k=\mathbb{Q}$, a set projectively equivalent to

$$
\mathbb{Z}_{(p)} \subset \mathbb{A}^{1}(\mathbb{Q}) \subset \mathbb{P}^{1}(\mathbb{Q})
$$

the set of rational numbers with denominator coprime to $p$.

Indeed, such a subgroup is necessarily either $F^{\times} / k^{\times}$for some subfield $F \subset K$, or $\mathfrak{o}_{K, \nu}$, for some valuation $\nu$ (see Section 7). By construction, the homomorphism $\psi$ will satisfy the cases $(\mathrm{P})$ or $(\mathrm{V})$ in Theorem 2 , respectively.

To find such $\mathfrak{U}$, we use results of [6] and [1]. First we deduce that the restriction of $\psi$ to every plane $\mathbb{P}^{2} \subset \mathbb{P}(K)$ is either an embedding or is induced by a natural construction from some nonarchimedean valuation (see Section 5). We distinquish two cases:

- there exists a line $\mathfrak{l} \subset \mathbb{P}(K)$ such that the restriction of $\psi$ to $\mathfrak{l}$ is injective,

- no such lines exist.

In the first case, property (4) of Condition 3 does not occur, and the proof works uniformly for $k=\mathbb{F}_{p}$ or $\mathbb{Q}$. In the second case, the proofs are slightly different, leading to a case-by-case analysis in Section 5 .

Acknowledgments. This work was partially supported by Laboratory of Mirror Symmetry NRU HSE, RF grant 14.641.31.0001. The first and second authors were funded by the Russian Academic Excellence Project '5-100'. The first author was also supported by a Simons Fellowship and by the EPSRC program grant EP/M024830. The third author was partially supported by the NSF grant 1601912.

\section{Projective Geometry}

Let $\mathbb{P}$ be a projective space over a field $k$ and $\Pi\left(\mathfrak{q}_{0}, \ldots, \mathfrak{q}_{n}\right) \subseteq \mathbb{P}$ the projective envelope of points $\mathfrak{q}_{0}, \ldots, \mathfrak{q}_{n} \in \mathbb{P}$. Working with lines and planes, we write

$$
\mathfrak{l}=\mathfrak{l}\left(\mathfrak{q}_{0}, \mathfrak{q}_{1}\right), \quad \text { resp. } \quad \Pi=\Pi\left(\mathfrak{q}_{0}, \mathfrak{q}_{1}, \mathfrak{a}_{2}\right),
$$

for a projective line through $\mathfrak{q}_{0}, \mathfrak{q}_{1}$, or a plane through $\mathfrak{q}_{0}, \mathfrak{q}_{1}, \mathfrak{q}_{2}$. 
Let $\nu$ a nonarchimedean valuation of $k, \mathfrak{o}=\mathfrak{o}_{\nu}$ the corresponding valuation ring, and $\boldsymbol{k}_{\nu}$ the residue field. Fixing a lattice

$$
\Lambda \simeq \mathfrak{o}^{n+1} \hookrightarrow k^{n+1}
$$

we obtain a natural surjection

$$
\rho=\rho_{\Lambda}: \mathbb{P}^{n}(k) \rightarrow \mathbb{P}^{n}\left(\boldsymbol{k}_{\nu}\right) .
$$

A 3-coloring of $\mathbb{P}^{2}(k)$ is a surjection

$$
\mathfrak{c}: \mathbb{P}^{2}(k) \rightarrow\{\bullet, \circ, \star\},
$$

onto a set of 3 elements, such that

- every $\mathfrak{l} \subset \mathbb{P}^{2}(k)$ is colored in exactly two colors, i.e., $\mathfrak{c}(\mathfrak{l})$ consists of two elements.

A 3-coloring is called trivial of type

- $I$ : if there exists a line $\mathfrak{l} \subset \mathbb{P}^{2}$ such that $\mathfrak{c}$ is constant on $\mathbb{P}^{2} \backslash \mathfrak{l}$,

- $I I$ : if there exists a point $\mathfrak{q} \in \mathbb{P}^{2}(k)$ such that for every $\mathfrak{l} \subset \mathbb{P}^{2}$ containing $\mathfrak{q}, \mathfrak{c}$ is constant on $\mathfrak{l} \backslash \mathfrak{q}$.

It was discovered early on, that such colorings are related to valuations, see, e.g., [6]. The same structure resurfaced in the study of commuting elements of Galois groups of function fields in [2], exhibiting unexpected projective structures within $\mathcal{G}_{K}^{a}$. This was a crucial step in the recognition of inertia and decomposition subgroups in $\mathcal{G}_{K}^{a}$.

Precisely, we have (see [6, Theorem 2] and [2]):

Proposition 4. Assume that $\mathbb{P}^{2}(k)$ carries a 3-coloring. Then there exists a nonarchimedean valuation $\nu$ such that the coloring $\mathfrak{c}$ in (7) is induced from a trivial covering

$$
\mathfrak{c}_{\nu}: \mathbb{P}^{2}\left(\boldsymbol{k}_{\nu}\right) \rightarrow\{\bullet, \circ, \star\}
$$

for some $\rho$ as in (6).

\section{FLAG MAPS}

We will consider maps (respectively, homomorphisms)

$$
f: \mathbb{P} \rightarrow A
$$

from projective spaces over $k$ to a set (respectively, an abelian group). The map $f$ is called a flag map if its restriction $f_{\Pi}$ to every finite dimensional projective subspace $\Pi \subset \mathbb{P}$ is a flag map. For $k=\mathbb{F}_{p}$ and

$$
f: \mathbb{P}^{n}\left(\mathbb{F}_{p}\right) \rightarrow A,
$$

this means that there exists a flag of projective subspaces

$$
\mathbb{P}^{n} \supset \mathbb{P}^{n-1} \ldots \supset \mathbb{P}^{1} \supset \mathbb{P}^{0}=\mathfrak{q}
$$


such that $f$ is constant on $\mathbb{P}^{i}\left(\mathbb{F}_{p}\right) \backslash \mathbb{P}^{i-1}\left(\mathbb{F}_{p}\right)$, for all $i=1, \ldots, n$. For $k=\mathbb{Q}$ and

$$
f: \mathbb{P}^{n}(\mathbb{Q}) \rightarrow A
$$

this means that either

- there is a flag as in (8) so that $f$ is constant on $\mathbb{P}^{i}(\mathbb{Q}) \backslash \mathbb{P}^{i-1}(\mathbb{Q})$, for all $i=1, \ldots, n$, or

- there exist a prime $p$, a surjection

$$
\rho=\rho_{\Lambda}: \mathbb{P}^{n}(\mathbb{Q}) \rightarrow \mathbb{P}^{n}\left(\mathbb{F}_{p}\right)
$$

as in ([6), and a flag map

$$
\bar{f}: \mathbb{P}^{n}\left(\mathbb{F}_{p}\right) \rightarrow A
$$

such that

$$
f=\bar{f} \circ \rho .
$$

Proposition 5. 2] Let

$$
f: \mathbb{P}(K)=K^{\times} / k^{\times} \rightarrow A
$$

be a group homomorphism which is also a flag map. Then there exist a valuation $\nu$ of $K$ and a homomorphism $r: \Gamma_{\nu} \rightarrow A$ such that $f$ factors through

$$
K^{\times} / k^{\times} \stackrel{\nu}{\longrightarrow} \Gamma_{\nu} \stackrel{r}{\longrightarrow} A .
$$

A flag map $f$ on $\mathbb{P}^{n}(k)$ defines a map

$$
\begin{aligned}
\hat{f}: \hat{\mathbb{P}}^{n} & \rightarrow A \\
\lambda & \mapsto f_{\text {gen }}(\lambda)
\end{aligned}
$$

on the dual space, by assigning to a projective hyperplane the generic value of $f$ on this hyperplane. The following lemma generalizes results in [2, Section 2].

Lemma 6. A map $f: \mathbb{P} \rightarrow A$ is a flag map if and only if for every $\Pi=\mathbb{P}^{2}(k) \subset \mathbb{P}$ the restriction $f_{\Pi}$ is a flag map.

Proof. Assume the claim for every $\mathbb{P}^{n-1} \subset \mathbb{P}^{n}$, for $n \geq 3$.

Step 1. Consider $\mathfrak{q}_{1}, \ldots, \mathfrak{q}_{r} \in \mathbb{P}^{n}(k)$ so that $f\left(\mathfrak{q}_{i}\right)$ are not generic in $\mathbb{P}^{n}$ and let $\Pi=\Pi\left(\mathfrak{q}_{1}, \ldots, \mathfrak{q}_{r}\right) \subset \mathbb{P}^{n}(k)$ be their projective envelope. If $\operatorname{dim}(\Pi) \geq n-1$ then, for some $i$,

$$
f\left(\mathfrak{q}_{i}\right)=f_{\text {gen }}(\Pi)
$$

Indeed, if $\mathfrak{q}_{1}, \ldots, \mathfrak{q}_{r}$ are nongeneric then any subset generates a subspace of dimension $\leq n-2$ and hence $\left\{\mathfrak{q}_{1}, \ldots, \mathfrak{q}_{r}\right\} \subset \Pi, \operatorname{dim}(\Pi) \leq n-2$, by induction. Then $f$ is constant outside of $\Pi$ and hence, by induction, a flag map. Thus we may assume $\operatorname{dim}(\Pi)<n-1$. 
Step 2. $\hat{f}$ takes at most two values. Indeed, assume it takes distinct values $a_{i}$ on hyperplanes $\mathbb{P}_{i}^{n-1}, i=1,2,3$, so that $f$ is constant on affine subspaces $\mathbb{A}_{i}^{n-1} \subset \mathbb{P}_{i}^{n-1}$. There exists a $\mathfrak{q}_{3} \in \mathbb{A}_{3}^{n-1}$, not contained in $\mathbb{P}_{1}^{n-1} \cup \mathbb{P}_{2}^{n-1}$, since $\mathbb{A}_{1}^{n-1}, \mathbb{A}_{2}^{n-1}, \mathbb{A}_{3}^{n-1}$ are disjoint and

$$
\left(\mathbb{P}_{1}^{n-1} \cup \mathbb{P}_{2}^{n-1}\right) \backslash\left(\mathbb{A}_{1}^{n-1} \cup \mathbb{A}_{2}^{n-1}\right)=\mathbb{P}^{n-2}=\mathbb{P}_{1}^{n-1} \cap \mathbb{P}_{2}^{n-1},
$$

which does not contain an affine space $\mathbb{A}^{n-1}$. Consider the projection

$$
\pi_{3}: \mathbb{P}^{n} \backslash\left\{\mathfrak{q}_{3}\right\} \rightarrow \mathbb{P}^{n-1}
$$

from $\mathfrak{q}_{3}$. Then there exists a point

$$
\mathfrak{q} \subset \pi_{3}\left(\mathbb{A}_{1}^{n-1}\right) \cap \pi_{3}\left(\mathbb{A}_{2}^{n-1}\right) \subset \mathbb{P}^{n-1} .
$$

The restriction of $f$ to $\mathfrak{l}\left(\mathfrak{q}_{3}, \mathfrak{q}\right)$ takes three values, and $f$ is not a flag map on $\mathbb{P}^{2}$ containing this line, contradiction.

Step 3. Consider $\mathbb{P}_{1}^{n-1}$ with generic value $a_{1}$ and let $\mathfrak{q} \notin \mathbb{P}_{1}^{n-1}$ be a point $f(\mathfrak{q}) \neq a_{2}$. Let $\mathbb{P}^{\prime} \subset \mathbb{P}_{1}^{n-1}$ be a maximal projective subspace with a generic value different from $a_{1}$. On any $\mathbb{P}_{\mathfrak{q}}^{n-1} \subset \mathbb{P}^{n}$ containing $\mathfrak{q}$ and such that $\mathbb{P}_{\mathfrak{q}}^{n-1} \cap \mathbb{P}_{1}^{n-1} \neq \mathbb{P}^{\prime}$ the generic value $\hat{f}_{\mathfrak{q}}=a_{1}$. Indeed, it is generated by $\mathfrak{q}$ and points of $\mathbb{P}_{1}^{n-1} \backslash \mathbb{P}^{\prime}$. Thus $\hat{f}_{\mathfrak{q}} \neq a_{2}$, and since $f$ takes only two values, $\hat{f}_{\mathfrak{q}}=a_{1}$. In particular, on $\hat{\mathbb{P}}_{\mathfrak{q}}^{n-1} \subset \hat{\mathbb{P}}^{n}$, which corresponds to all hyperplanes containing $\mathfrak{q}$, there is at most one point with $\hat{f} \neq 1$. This can only occur if $\operatorname{dim}\left(\mathbb{P}^{\prime}\right)=n-1$.

The same argument holds for $\mathbb{P}_{2}^{n-1}$ and the corresponding subspace $\hat{\mathbb{P}}_{u}^{n-1} \subset \hat{\mathbb{P}}^{n}$. Since $\hat{\mathbb{P}}_{\mathfrak{q}}^{n-1}$ and $\hat{\mathbb{P}}_{u}^{n-1}$ intersect in $\mathbb{P}^{n-2}$ we obtain a contradiction, unless all the points with value $a_{i}$ are contained in a hyperplane (for $i=1$ or 2 ), if $n>2$. Thus $f$ is a flag map.

The following lemma is in [1, Lemma 5.2] (corrected in [2, Prop. 3.4.1]).

Lemma 7. Let $k=\mathbb{Q}$ or $k=\mathbb{F}_{p}$, with $p>2$, and let

$$
f: \mathbb{P}^{2}(k) \rightarrow A
$$

be such that for every line $\mathfrak{l} \subset \mathbb{P}^{2}$ the restriction $f_{\mathfrak{l}}$ is a flag map. Then $f$ is a flag map.

Proof. The proof of Lemma 6 works up to Step 2. Thus $\hat{f}$ takes two values $a_{1}, a_{2}$ and either

(1) there is exactly one point $\mathfrak{q}$ with $f(\mathfrak{q})=a_{3}$ or

(2) $\hat{f}$ takes two values.

In the first case, we apply Proposition 4, In the second case, either

- one of the values is concentrated on a line or 
- there exist $x_{1}, x_{2}, x_{3}$ and $y_{1}, y_{2}, y_{3}$ spanning $\mathbb{P}^{2}(k)$ with $f\left(x_{i}\right)=$ $a_{1}$ and $f\left(y_{i}\right)=a_{2}$.

Consider the projection

$$
h_{x_{1}}: \mathbb{P}^{2} \backslash x_{1} \rightarrow \mathfrak{l}:=\mathfrak{l}\left(x_{2}, x_{3}\right) .
$$

The generic value on $\mathfrak{l}$ is $a_{1}$ and hence there is at most one $\mathfrak{q}_{2} \in \mathfrak{l}$ with $f\left(\mathfrak{q}_{2}\right)=a_{2}$. The generic value of $f$ on any $\mathfrak{l}\left(x_{1}, x\right), x \in \mathfrak{l} \backslash \mathfrak{q}_{2}$ is $a_{1}$. Over $k=\mathbb{F}_{p}$, the number of points with $f=a_{2}$ is $\leq 2 p$. The same argument applies for $y_{1}$, and it follows

$$
\mathbb{P}^{2}\left(\mathbb{F}_{p}\right)=p^{2}+p+1 \leq 4 p, \quad \text { i.e., } p=2 .
$$

This approach works also over $k=\mathbb{Q}$.

Remark 8. Lemma 7 fails over $\mathbb{F}_{2}$, since any map with two values on $\mathbb{P}^{1}\left(\mathbb{F}_{2}\right)$ is a flag map.

Lemma 9. Let

$$
f: \mathbb{P}(K)=K^{\times} / k^{\times} \rightarrow A
$$

be a group homomorphism whose restriction to every line is a flag map, and such that there exists a plane $\Pi=\Pi(1, x, y)$, with $f(x), f(y) \neq 1$, and $f_{\Pi}$ not a flag map. Then

$$
f(x)=f(y) \quad \text { and } \quad f(x)^{2}=1 .
$$

In particular, if $f$ is not a flag map, then $k=\mathbb{F}_{2}$ and $f^{2}$ is a flag map.

Proof. By Lemma 7, $k=\mathbb{F}_{2}$, so that $K^{\times} / k^{\times}=K^{\times}$. Then $f_{\Pi}$ takes two values and is constant, with distinct values, on two triples of noncollinear points. Thus

(i) $f$ is constant on $\mathfrak{l} \cup\{\mathfrak{q}\}$, where $\mathfrak{l} \subset \Pi$ is a line (containing the remaining seventh point) and $\mathfrak{q} \in \Pi \backslash \mathfrak{l}$, and

(ii) $f$ is constant on $\left\{x_{1}, x_{2}, x_{3}\right\}:=\Pi \backslash(\mathfrak{l} \cup\{\mathfrak{q}\})$; put $a:=f\left(x_{i}\right)$.

After a shift, we may assume that $\mathfrak{q}=1$, so $f(\mathfrak{l})=1$. Suppose that $a^{2} \neq 1$. Let $\mathbb{P}_{i}^{3}$ be the projective envelope of $\Pi \cup\left\{x_{i}^{2}\right\}$. We claim that $x_{i}^{2} \in \mathbb{P}_{i}^{3}$ is the only point with $f\left(x_{i}^{2}\right)=a^{2}$, in particular, $f$ is constant, on the complement to $x_{i}^{2}$, on lines in $\mathbb{P}_{i}^{3}$ passing through $x_{i}^{2}$.

Note that $f$ takes three values on $\mathbb{P}_{i}^{2}:=\Pi\left(1, x_{i}, x_{i}^{2}\right)$, and thus is a flag map on $\mathbb{P}_{i}^{2}$. Let $y_{i}=x_{i}+1$ be the only point of $\mathfrak{l}\left(1, x_{i}\right) \backslash\left\{1, x_{i}\right\}$. Then $f\left(y_{i}\right)=1$, since $y_{i} \in \mathfrak{l}$. Thus, $f(1)=f\left(y_{i}\right)=f\left(y_{i}^{2}\right)=1$ and $f\left(x_{i}\right)=f\left(x_{i} y_{i}\right)=a$ and $f\left(x_{i}^{2}\right)=a^{2}$, so 1 is the $f$-generic value on $\mathbb{P}_{i}^{2}$, and therefore, the remaining point

$$
\mathfrak{q}_{i}=x_{i}^{2}+x_{i}+1 \in \mathbb{P}_{i}^{2} \backslash\left(\mathfrak{l}\left(1, x_{i}\right) \cup \mathfrak{l}\left(1, x_{i}^{2}\right) \cup \mathfrak{l}\left(x_{i}, x_{i}^{2}\right)\right)
$$

is also $f$-generic, i.e., $f\left(\mathfrak{q}_{i}\right)=1$. 
We have $4=2^{3}-2^{2}$ points in $\mathbb{P}_{i}^{3} \backslash\left(\Pi \cup \mathbb{P}_{i}^{2}\right)$, where $f$ is not yet determined. They are contained, for $r \neq i$, in $\Pi\left(1, x_{i}, x_{i}^{2}+x_{r}\right) \backslash \mathfrak{l}\left(1, x_{i}\right)$, which consists of

$$
x_{i}^{2}+x_{r}, \quad x_{i}^{2}+x_{j}, \quad x_{i}^{2}+x_{j}+1, \quad x_{i}^{2}+x_{r}+1 .
$$

Each of these is contained in a line intersecting $\Pi$ and $\mathbb{P}_{i}^{2}$ at points with different values $a$ and 1 . Indeed, for $\{i, j, r\}=\{1,2,3\}$, one has

- $x_{i}^{2}+x_{r} \in \mathfrak{l}\left(q_{i}, x_{j}\right) \cap \mathfrak{l}\left(x_{i}^{2}, x_{r}\right)$, so

$$
f\left(x_{i}^{2}+x_{r}\right) \in\{1, a\} \cap\left\{a^{2}, a\right\}=\{a\} ;
$$

- $x_{i}^{2}+y_{r} \in \mathfrak{l}\left(x_{i}^{2}+1, x_{r}\right) \cap \mathfrak{l}\left(x_{i}^{2}, y_{r}\right)$, and

$$
f\left(x_{i}^{2}+y_{r}\right) \in\{1, a\} \cap\left\{a^{2}, 1\right\}=\{1\} .
$$

Note that $f$ takes three values on $\mathfrak{l}\left(x_{1}^{2}, x_{2}^{2}+x_{1}\right)$ :

$$
f\left(x_{1}^{2}\right)=a^{2}, \quad f\left(x_{2}^{2}+x_{1}\right)=a, \quad f\left(x_{3}^{2}+x_{1}+1\right)=1 .
$$

If $a^{2} \neq 1$ we get a contradiction to our assumption that $f$ takes only two values and is flag on any line in $\Pi\left(x_{1}, x_{2}, x_{3}\right)$. Thus on every $\Pi(1, x, y)$ where $f$ is not a flag map, $f^{2} \equiv 1$, hence is a flag map.

Remark 10. Under conditions of Lemma 7, $f^{2}$ is always a flag map. In particular, if $A$ has no 2-torsion, then Proposition 5 holds as well.

Lemma 11. Assume the conditions of Lemma 7 and that the twotorsion part $A[2] \subseteq A$ is nontrivial. Consider the composition

$$
f_{2}: \mathbb{P}(K) \stackrel{f}{\longrightarrow} A \stackrel{r_{2}}{\longrightarrow} A / A[2],
$$

with $r_{2}$ the projection. Then $f_{2}$ is a flag map on every plane $\Pi \subset \mathbb{P}(K)$.

Proof. If $f$ is a flag map on $\Pi(1, x, y)$ then $f_{2}$ is also flag. If $f$ is not a flag map, then we apply Lemma 9, $f$ takes only two values 0 or 1 , and $f(x)^{2}=1$, thus $f(x)=1$.

In particular, $f_{2} \equiv 1$ on $\Pi(1, x, y)$ and hence is a flag map. Thus $f_{2}$ is a flag map on every plane and hence a flag map.

To summarize, if $A \neq A[2]$ then $f$ determines a valuation $\nu$. If $A=A[2]$, then $f$ is trivial on some subfield $K^{\prime} \subset K$ such that $K \mid K^{\prime}$ is a purely inseparable extension of exponent 2 .

\section{3. $\mathbb{Z}_{(p)}$-LATTICES}

Let $p$ be a prime number and $\mathbb{Z}_{(p)} \subset \mathbb{Q}$ the set of rational numbers with denominator coprime to $p$. A $\mathbb{Z}_{(p)}$-lattice, or simply, a lattice

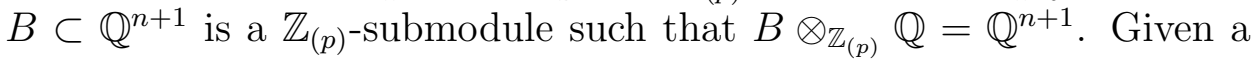
lattice $B \subset \mathbb{Q}^{n+1}$ and an element $x \in \mathbb{Q}^{n+1} \backslash 0$ there exists an element 
$x_{B} \in B \backslash p B$ such that $x$ and $x_{B}$ define the same point in $\mathbb{P}^{n}(\mathbb{Q})$, this element is unique in $B \backslash p B$, modulo scalar multiplication by $\mathbb{Z}_{(p)}^{\times}$. Lattices $B, B^{\prime} \subset \mathbb{Q}^{n+1}$ are called equivalent if $B=a \cdot B^{\prime}$, for some $a \in \mathbb{Q}^{\times}$.

In this section, we consider maps

$$
f:\left(\mathbb{Q}^{n+1} \backslash 0\right) \rightarrow A
$$

which are invariant under scalar multiplication by $\mathbb{Q}^{\times}$; we use the same notation for the induced map

$$
f: \mathbb{P}^{n}(\mathbb{Q}) \rightarrow A .
$$

We say that $f$ is induced from $\mathbb{P}^{n}(\mathbb{Z} / p)$ via a lattice $B$ if there exists a map

$$
\bar{f}: \mathbb{P}^{n}(\mathbb{Z} / p) \rightarrow A
$$

such that

$$
f(x)=\left(\bar{f} \circ \rho_{B}\right)\left(x_{B}\right), \quad \text { for all } \quad x \in \mathbb{P}^{n}(\mathbb{Q}),
$$

where

$$
\rho_{B}:(B \backslash p B) \rightarrow(B / p B) \backslash 0 \rightarrow \mathbb{P}^{n}(\mathbb{Z} / p) .
$$

This is well-defined since $\rho_{B}$ is invariant under $\mathbb{Z}_{(p)}^{\times}$. Such lattices will be called $f$-compatible, or simply compatible. If $f$ is induced from $\mathbb{P}^{n}(\mathbb{Z} / p)$ via a lattice $B$ then it is also induced via any equivalent lattice.

Lemma 12. Assume that $f$ is induced from $\mathbb{P}^{n}(\mathbb{Z} / p)$ via a lattice $B$. Let $\bar{x} \in \mathbb{P}^{n}(\mathbb{Z} / p)$ and choose an $x_{B} \in B \backslash p B$ such that $\rho_{B}\left(x_{B}\right)=\bar{x}$. Let

$$
N_{\bar{x}, B}:=\left\{x \in \mathbb{Q}^{n+1} \backslash 0 \mid x=x_{B} \in \mathbb{P}^{n}(\mathbb{Q})\right\} .
$$

If $B^{\prime}$ is another $f$-compatible lattice such that

$$
N_{\bar{x}^{\prime}, B^{\prime}}=N_{\bar{x}, B}, \quad \text { for some } \quad \bar{x}^{\prime} \in \mathbb{P}^{n}(\mathbb{Z} / p),
$$

then $B^{\prime}$ is equivalent to $B$.

Proof. We have

$$
N_{\bar{x}, B}=\mathbb{Q}^{\times} \cdot\left(x_{B}+p B\right), \quad x_{B} \in(B \backslash p B), \quad \rho_{B}\left(x_{B}\right)=\bar{x} .
$$

Consider $z \in\left(\mathbb{Q}^{n+1} \backslash 0\right) \backslash N_{\bar{x}, B}$ such that $x_{B}+z$ projects to $\bar{x} \in \mathbb{P}^{n}(\mathbb{Z} / p)$. Note that $z \notin(B \backslash p B)$ since otherwise $\overline{x+z} \neq \bar{x}$. Furthermore, $z \notin \mathbb{Q}^{n+1} \backslash B$, since otherwise $z \in p^{-m}(B \backslash p B)$, for some $m \in \mathbb{N}$, and $\overline{x_{B}+z}=\bar{z} \neq \bar{x}$. Thus $z \in p B$, and the lattice $B_{x}$ spanned by such $z$ equals $p B$. Hence for any $y \in N_{\bar{x}, B}, B_{y}=p^{m} B$, for some $m \in \mathbb{Z}$.

In the discussion below, we use projective and affine geometry. The following lemma connects these concepts. 
Lemma 13. Let $L=\mathbb{A}^{2}\left(\mathbb{Z}_{(p)}\right) \subset \mathbb{A}^{2}(\mathbb{Q}) \subset \mathbb{P}^{2}(\mathbb{Q})$ and

$$
\rho_{L}: L \rightarrow \bar{L}=\mathbb{A}^{2}(\mathbb{Z} / p)
$$

be the reduction map. Then there exists a unique equivalence class of $\mathbb{Z}_{(p)}$-lattices $B \subset \mathbb{Q}^{3}$ such that $\rho$ extends to

$$
\rho_{B}: B \backslash p B \rightarrow \mathbb{P}^{2}(\mathbb{Z} / p)
$$

and such that the corresponding map $\mathbb{P}^{2}(\mathbb{Q}) \rightarrow \mathbb{P}^{2}(\mathbb{Z} / p)$ coincides with $\rho_{L}$ upon restriction to $L$.

Proof. Let $\tilde{x} \in \mathbb{Q}^{3}$ be an element projecting to $x \in L \subset \mathbb{P}^{2}(\mathbb{Q})$. Consider a subspace $\mathbb{Q}^{2} \subset \mathbb{Q}^{3}$ such that the corresponding line $\mathfrak{l} \subset \mathbb{P}^{2}$ is disjoint from $L$. Then the preimage of $L$ in $x+\mathbb{Q}^{2} \subset \mathbb{Q}^{3}$ coincides with the $x+B^{\prime}$, for some lattice $B^{\prime} \subset \mathbb{Q}^{3}$, and the lattice generated by $x$ and $p B^{\prime}$ is the desired lattice $B$. Indeed, $x+p B^{\prime}$ is the preimage of a point in $\bar{L}$, hence the sublattice $B$ defines a map $\rho_{B}$ with desired properties; all such lattice are equivalent, by Lemma 12 .

Lemma 14. Assume that $f: \mathbb{P}^{1}(\mathbb{Q}) \rightarrow A$ is induced from a nonconstant map $\bar{f}: \mathbb{P}^{1}(\mathbb{Z} / p) \rightarrow A$, via some lattice.

(1) If $\bar{f}$ is flag map, then there are exactly two equivalence classes of $f$-compatible lattices $B_{1}, B_{2} \subset \mathbb{Q}^{2}$.

(2) If $\bar{f}$ is not a flag map, then there is exactly one equivalence class of $f$-compatible lattices $B \subset \mathbb{Q}^{2}$.

Proof. By assumption, $f$ is induced via some $\rho_{B}$. Fix generators $x, y \in$ $B$ such that $f(y) \neq f(x)$, in particular $\rho_{B}\left(x_{B}\right) \neq \rho_{B}\left(y_{B}\right) \in \mathbb{P}^{1}(\mathbb{Z} / p)$. We have

$$
f(y+p B)=f(y) \text { and } f(x+p B)=f(x) \neq f(y) .
$$

Any lattice $B^{\prime} \subset \mathbb{Q}^{2}$ is equivalent to a lattice with $x$ as a generator. Since $B^{\prime} / \mathbb{Z}_{(p)} \cdot x \simeq \mathbb{Z}_{(p)}, B^{\prime}$ is one of the following: $B_{i}:=\left\langle x, p^{i} y\right\rangle$, for some $i \in \mathbb{Z}$. If $f$ is induced from $B_{i}$, for some $i<-1$, then

$f\left(x+p\left(p^{i} y\right)\right)=f(x) \neq f(y)$ and $f\left(x+p\left(p^{i} y\right)\right)=f\left(p^{-i-1} x+y\right)=f(y)$, a contradiction. The same argument gives a contractions when $i>1$. Thus $i=1,0$, or -1 .

Analysis of values of $\bar{f}$ at other points of $\mathbb{P}^{1}(\mathbb{Z} / p)$ leads to further restrictions. We have the following cases:

(1) $\bar{f}$ is constant on $\mathbb{P}^{1}(\mathbb{Z} / p) \backslash \rho_{B}\left(y_{B}\right)$.

(2) $\bar{f}$ is not constant on the complement to a point in $\mathbb{P}^{1}(\mathbb{Z} / p)$.

In Case (1), $f(x+y)=f(x)$, excluding $i=1$. Then we have exactly two lattices $B_{0}, B_{-1}$ such that $f$ is induced from these (or equivalent) lattices. 
In Case (2), if $f$ is induced from $B_{-1}$ then $f(\kappa x+y)=f(y)$, for any $\kappa \in \mathbb{Q}$, and hence $\bar{f}$ is constant on $\mathbb{P}^{1}(\mathbb{Z} / p) \backslash \rho_{B}\left(x_{B}\right)$, contradicting the second condition. Thus there is only one compatible lattice $B_{0}=B$, modulo equivalence.

Lemma 15. Assume that $f: \mathbb{P}^{2}(\mathbb{Q}) \rightarrow$ A satisfies the following:

(1) $f$ takes three values;

(2) $f$ takes at most two values on every line $\mathfrak{l} \subset \mathbb{P}^{2}$;

(3) on every $\mathbb{P}^{1}(\mathbb{Q}) \subset \mathbb{P}^{2}(\mathbb{Q})$, $f$ is induced from a flag map on $\mathbb{P}^{1}(\mathbb{Z} / p)$, via $\rho_{B^{\prime}}$, for some lattice $B^{\prime} \subset \mathbb{Q}^{2}$.

Then there are exactly three equivalence classes of lattices $B_{i} \subset \mathbb{Q}^{3}$ such that $f$ is induced from a flag map $\bar{f}: \mathbb{P}^{2}(\mathbb{Z} / p) \rightarrow A$, via $\rho_{B_{i}}, i=1,2,3$.

Proof. Follows from Proposition 4, applied to $k=\mathbb{Q}$ (see also [6] or [2]). The first two conditions imply that there exists a lattice $B \subset \mathbb{Q}^{3}$ such that $f$ is induced from some map $\bar{f}: \mathbb{P}^{2}(\mathbb{Z} / p) \rightarrow A$, via $B$. Applying both statements of Lemma 14, we conclude that $\bar{f}$ is a flag map, with 3 distinct values. Hence

$$
\mathbb{P}^{2}(\mathbb{Q})=S_{1} \sqcup S_{2} \sqcup S_{3},
$$

with $S_{1}$ the preimage of an affine plane in $\mathbb{P}^{2}(\mathbb{Z} / p), S_{2}$ an affine line, and $S_{3}$ a point in $\mathbb{P}^{2}(\mathbb{Z} / p)$, and $f$ is constant on these sets.

By Lemma 14, for any $B^{\prime} \subset \mathbb{Q}^{3}$ such that $f$ is induced from $\mathbb{P}^{2}(\mathbb{Z} / p)$ via $\rho_{B^{\prime}}$, the restriction of $f$ to any $\left(\mathbb{Q}^{2} \backslash 0\right) \subset\left(\mathbb{Q}^{3} \backslash 0\right)$ is induced from a flag map on $\mathbb{P}^{1}(\mathbb{Z} / p)$.

Thus $f$ is also induced from a flag map, via $\rho_{B^{\prime}}$. On the other hand, in coordinates $x_{1}, x_{2}, x_{3}$, we have

$S_{1}=\left\{x_{1} \neq 0\right\}, \quad S_{2}=\left\{x_{1}=0, x_{2} \neq 0\right\}, \quad S_{3}=\left\{x_{1}=x_{2}=0, x_{3} \neq 0\right\}$, and the only possible coordinates compatible with the structures on all $\mathbb{P}^{1}(\mathbb{Q})$ are

$$
x_{1}, \frac{x_{2}}{p}, \frac{x_{3}}{p}, \quad x_{1}, x_{2}, \frac{x_{3}}{p} \text {, and } x_{1}, x_{2}, x_{3} .
$$

This gives exactly three equivalence classes of $f$-compatible lattices.

Corollary 16. Assume that we are in the situation of Lemma 15 so that $f$ is induced from a flag map

$$
\bar{f}: \mathbb{P}^{2}(\mathbb{Z} / p) \rightarrow\{1,2,3\} .
$$

Let $\mathfrak{q} \subset \mathfrak{l} \subset \mathbb{P}^{2}(\mathbb{Z} / p)$ be the corresponding flag. Let $B^{\prime}$ be another $f$-compatible lattice,

$$
\bar{f}^{\prime}: \mathbb{P}^{2}(\mathbb{Z} / p) \rightarrow\{1,2,3\}
$$


the corresponding flag map inducing $f$, and $\mathfrak{q}^{\prime} \subset \mathfrak{l}^{\prime} \subset \mathbb{P}^{2}(\mathbb{Z} / p)$ the associated flag. If $\bar{f}(\mathfrak{q})=\bar{f}^{\prime}\left(\mathfrak{q}^{\prime}\right)$ then $B$ and $B^{\prime}$ are equivalent and $\bar{f}=\bar{f}^{\prime}$.

Thus if $f: \mathbb{P}^{1}(\mathbb{Q}) \rightarrow A$ is induced from $\bar{f}: \mathbb{P}^{1}(\mathbb{Z} / p) \rightarrow A$ then one of the following holds:

(1) The map $\rho: \mathbb{P}^{1}(\mathbb{Q}) \rightarrow \mathbb{P}^{1}(\mathbb{Z} / p)$ inducing $f$ is unique; in particular, for any $\mathfrak{q} \in \mathbb{P}^{1}(\mathbb{Z} / p)$ the subset $L_{p}:=\rho^{-1}\left(\mathbb{P}^{1}(\mathbb{Z} / p) \backslash \mathfrak{q}\right)$ is affinely isomorphic to $\mathbb{Z}_{(p)} \subset \mathbb{Q} \subset \mathbb{P}^{1}(\mathbb{Q})$ and the map $L_{p} \rightarrow \mathbb{Z} / p$ is uniquely defined as a linear map

$$
\begin{array}{clll}
\mathbb{Z}_{(p)} & \rightarrow & \mathbb{Z} / p \\
x & \mapsto & x & (\bmod p) .
\end{array}
$$

(2) $f$ is a flag map and there are two possible $\rho: \mathbb{P}^{1}(\mathbb{Q}) \rightarrow \mathbb{P}^{1}(\mathbb{Z} / p)$ inducing $f$.

\section{BASIC FIELD THEORY}

Let

$$
k \subseteq \tilde{k} \subseteq \tilde{k}_{a} \subseteq K
$$

be an extension of fields. We say that $x_{1}, x_{2} \in K^{\times} / k^{\times}$are algebraically dependent with respect to $\tilde{k}$ if they satisfy Equation (4) from the Introduction; in this case, we write $x_{1} \sim_{\tilde{k}} x_{2}$, or simply $x_{1} \sim x_{2}$. We record the following obvious properties of this equivalence relation:

(AI) If $x_{1} \sim_{\tilde{k}} x_{2}, x_{1} / x_{2} \notin \tilde{k}_{a}^{\times} / k^{\times}$, and $x \chi_{\tilde{k}} x_{1}$ then $x_{1} / x \chi_{\tilde{k}} x_{2} / x$.

(AG) The set of nonconstant algebraically dependent elements, together with $\left(\tilde{k}_{a}^{\times} / k^{\times}\right)$forms a subgroup of $K^{\times} / k^{\times}$.

Lemma 17. Let $K \mid k$ and $L \mid l$ be field extensions and

$$
\psi: K^{\times} / k^{\times} \rightarrow L^{\times} / l^{\times}
$$

a homomorphism such that its restriction to $\mathfrak{o}_{K, \nu}^{\times} / \mathfrak{o}_{k, \nu}^{\times}$factors as

$$
\mathfrak{o}_{K, \nu}^{\times} / \mathfrak{o}_{k, \nu}^{\times} \longrightarrow \boldsymbol{K}_{\nu}^{\times} / \boldsymbol{k}_{\nu}^{\times} \stackrel{\psi_{\nu}}{\longrightarrow} L^{\times} / l^{\times} .
$$

Assume that $\psi_{\nu}$ preserves algebraic dependence with respect to $\boldsymbol{k}_{\nu}$ and $l$. Then $\psi$ also preserves algebraic dependence with respect to $k$ and $l$.

Proof. Let $k(x) \subset K$ be a purely transcendental extension and

$$
E=\overline{k(x)} \subset K
$$

its algebraic closure in $K$. We claim that the restriction of $\psi$ to $E^{\times} / k^{\times}$ preserves algebraic dependence. This is clear if $\psi$ is injective and preserves algebraic dependence. Now assume that $\psi$ is defined through a valuation $\nu$, i.e., as in (11). There are two cases: 
Case 1. $\nu\left(k^{\times}\right)=\nu\left(E^{\times}\right)$. Then

$$
E^{\times}=\mathfrak{o}_{E, \nu}^{\times} \cdot k^{\times} .
$$

Since $\psi_{\nu}$ preserves algebraic dependence with respect to $\boldsymbol{k}_{\nu}$ and $l$, the claim follows.

Case 2. $\nu\left(k^{\times}\right) \subsetneq \nu\left(E^{\times}\right)$. Then $\nu\left(E^{\times}\right) / \nu\left(k^{\times}\right)$has $\mathbb{Q}$-rank 1, i.e., for $y, z \in E^{\times}$with nonzero $\nu(y), \nu(z) \in \nu\left(E^{\times}\right) / \nu\left(k^{\times}\right)$there are nonzero $n_{y}, n_{z} \in \mathbb{Z}$ such that $n_{y} \nu(y)=n_{z} \nu(z)$. Indeed, $y, z$ define a finite algebraic extension $k_{y, z}(x) \mid k(x)$, hence $\nu$ is nontrivial on $k(x)$, and the group

$$
\nu\left(k_{y, z}(x)^{\times}\right) / \nu\left(k(x)^{\times}\right)
$$

is finite. Let $g \in k(x)^{\times}$be such that the image of $\nu(g)$ in $\nu\left(E^{\times}\right) / \nu\left(k^{\times}\right)$ is infinite. Then for any $\sum_{i=0}^{n} a_{i} g^{i}$, with $a_{i} \in k$,

$$
\nu\left(\sum_{i=0}^{n} a_{i} g^{i}\right)=\min _{i}\left(\nu\left(a_{i} g^{i}\right)\right),
$$

since none of the monomials $a_{i} g^{i}$ have the same value under $\nu$. Thus,

$$
\nu\left(k(g)^{\times}\right)=\nu\left(k^{\times}\right) \times\langle\nu(g)\rangle,
$$

The extensions $k_{y, z}(x) \mid k(x)$ and $k(x) \mid k(g)$ are finite, thus

$$
\nu\left(k_{y, z}(x)^{\times}\right) /\left(\nu\left(k^{\times}\right) \times\langle\nu(g)\rangle\right)
$$

is also finite, which implies the result for $\nu\left(E^{\times}\right)$. Since $\psi\left(k^{\times}\right)=1$, $\psi\left(k_{y, z}(x)^{\times}\right)$is the product of a finite group and $\mathbb{Z}$. In particular, $\psi\left(k_{y, z}(x)^{\times}\right)$consists of algebraically dependent elements. Since $E$ is a union of subfields $k_{y, z}(x)$, the same holds for $E^{\times}$.

Thus $\psi\left(E^{\times} / k^{\times}\right)$coincides with the image of $\nu\left(E^{\times}\right) / \nu\left(k^{\times}\right)$. Since all elements in $\nu\left(E^{\times}\right) / \nu\left(k^{\times}\right)$have the same powers we see that lifts of elements in $\psi\left(E^{\times}\right)$to $L^{\times}$are algebraically dependent over $l$.

\section{Restriction to PlAnes}

Here we study restrictions of homomorphisms

$$
\psi: \mathbb{P}(K)=K^{\times} / k^{\times} \rightarrow A:=L^{\times} / \tilde{l}^{\times},
$$

satisfying assumptions of Theorem 2 , to projective planes $\Pi \subset \mathbb{P}(K)$.

Proposition 18. Let $\Pi:=\Pi(1, x, y) \subset \mathbb{P}(K)$ be such that $\psi(x) \neq$ $\psi(y)$. Then one of the following holds:

(a) $\psi_{\Pi}$ is injective.

(b) There exists a line $\mathfrak{l} \subset \Pi$ such that $\psi_{\Pi}$ is constant on $\Pi \backslash \mathfrak{l}$. 
(c) There exists a point $\mathfrak{q} \in \Pi$ such that $\psi_{\Pi}$ is constant on $\mathfrak{l} \backslash \mathfrak{q}$, for every $\mathfrak{l} \subset \Pi$ passing through $\mathfrak{q}$.

(d) $k=\mathbb{Q}, \psi_{\Pi}$ is induced from

$$
\bar{\psi}_{\Pi}: \mathbb{P}^{2}(\mathbb{Z} / p) \rightarrow A,
$$

via a lattice $B \subset \mathbb{Q}^{3}$, and $\bar{\psi}_{\Pi}$ is of type $(a),(b)$, or $(c)$.

Proof. Assume that $\psi_{\Pi}$ is not injective: there are distinct $x_{1}, x_{2} \in \Pi$, with $\psi\left(x_{1}\right)=\psi\left(x_{2}\right) \neq 1$. Consider

$$
\Pi_{1}:=x_{1}^{-1} \cdot \Pi=\Pi\left(1,1 / x_{1}, y / x_{1}\right),
$$

since $\psi(y) \nsim \psi\left(1 / x_{1}\right), \Pi_{1}$ satisfies the conditions of the theorem; if it holds for $\Pi_{1}$ then it holds for the initial $\Pi$. Thus we may assume that

$$
S_{1}:=\left\{x^{\prime} \in \Pi \mid \psi\left(x^{\prime}\right)=1\right\}
$$

contains at least two elements. Consider the map

$$
\psi_{\sim}: \mathbb{P}(K) \rightarrow A_{\sim},
$$

with values in dependency classes:

- $\psi_{\sim}\left(x^{\prime}\right)=1$ if $\psi\left(x^{\prime}\right)=1$,

- $\psi_{\sim}\left(x^{\prime}\right)=\psi_{\sim}\left(x^{\prime \prime}\right)$ iff $\psi\left(x^{\prime}\right), \psi\left(x^{\prime \prime}\right) \neq 1$ and $\psi\left(x^{\prime}\right) \sim \psi\left(x^{\prime \prime}\right)$.

We record properties of $\psi_{\sim}$ :

(TI) For every $\mathfrak{l} \subset \Pi$ with $\mathfrak{l} \cap S_{1}=\emptyset$, we have

$$
\left\{\psi_{\sim}\left(x^{\prime}\right) \mid x^{\prime} \in \mathfrak{l}\right\}=\left\{\psi_{\sim}\left(x^{\prime \prime}\right) \mid x^{\prime \prime} \in \Pi \backslash S_{1}\right\},
$$

in particular, $\psi(\mathfrak{l})$ has has algebraically independent elements.

(TC) For every $\mathfrak{l} \subset \Pi$ with $\mathfrak{l} \cap S_{1} \neq \emptyset, \psi_{\sim}$ is constant on $\mathfrak{l} \backslash\left(\mathfrak{l} \cap S_{1}\right)$.

Property (AI) from Section 4 relates $\psi_{\sim}$ and $\psi$.

Lemma 19. If $\mathfrak{l} \cap S_{1}=\emptyset$ and $x^{\prime}, x^{\prime \prime} \in \mathfrak{l}$ are such that $\psi\left(x^{\prime}\right) \sim \psi\left(x^{\prime \prime}\right)$ then $\psi\left(x^{\prime}\right)=\psi\left(x^{\prime \prime}\right)$.

Proof. There is a $z \in \mathfrak{l}$ with $\psi(z) \not \psi\left(x^{\prime}\right), \psi\left(x^{\prime \prime}\right)$. Since $z^{-1} \cdot \mathfrak{l} \cap S_{1} \neq \emptyset$, all values of $\psi$ on $\mathfrak{l}\left(x^{\prime} / z, x^{\prime \prime} / z\right) \backslash 1$ are algebraically dependent. By (AI), if $\psi\left(x^{\prime}\right) \neq \psi\left(x^{\prime \prime}\right)$ then $\psi\left(x^{\prime}\right) / \psi(z) \nsim \psi\left(x^{\prime \prime}\right) / \psi(z)$, a contradiction.

Lemma 20. Let $\mathfrak{l}, \mathfrak{l}^{\prime} \subset \Pi$ be disjoint from $S_{1}$, put $z:=\mathfrak{l} \cap \mathfrak{l}^{\prime}$, and assume that there exist $x \in \mathfrak{l}$ and $x^{\prime} \in \mathfrak{l}^{\prime}$ such that

$$
\psi(x) \sim \psi\left(x^{\prime}\right), \psi(x) \neq \psi\left(x^{\prime}\right), \quad \text { and } \quad \psi(x), \psi\left(x^{\prime}\right) \nsim \psi(z) .
$$

Let $y \in \mathfrak{l}$ and $y^{\prime} \in \mathfrak{l}^{\prime}$ be such that $\psi(y) \sim \psi\left(y^{\prime}\right)$. Then either

- $\psi(y) \neq \psi\left(y^{\prime}\right)$, or

- $\psi(y)=\psi\left(y^{\prime}\right)=\psi(z)$. 
Proof. By the same argument as in Lemma 19, using (AI),

$$
\psi(x) / \psi(z) \sim \psi(y) / \psi(z), \quad \text { and } \psi\left(x^{\prime}\right) / \psi(z) \sim \psi\left(y^{\prime}\right) / \psi(z),
$$

If $\psi(y)=\psi\left(y^{\prime}\right) \neq \psi(z)$ then $\psi(x) / \psi(z) \sim \psi\left(x^{\prime}\right) / \psi(z)$, contradiction.

Let $\left\{T_{j}\right\}_{j \in J}$ be the set of intersections of algebraic dependency classes in $\mathbb{P}(K)$ with $\Pi$. Split $J=J_{2} \sqcup J_{3}$ and consider the decomposition

(12) $\Pi=S_{1} \sqcup S_{2} \sqcup S_{3}, \quad$ with $\quad S_{1}=T_{1}, S_{2}=\sqcup_{j \in J_{2}} T_{j}, \quad S_{3}=\sqcup_{j \in J_{3}} T_{j}$.

For any such decomposition, the induced map

$$
\Psi=\Psi_{\Pi}: \Pi \rightarrow\{1,2,3\}
$$

factors through $\psi_{\sim}$ and satisfies the conditions of Proposition 4. Thus $\Psi$ is induced from a trivial coloring, with $S_{1}$ not depending on the decomposition. Since there exist lines disjoint from $S_{1}$, and $S_{1}$ contains at least two points, it follows that either

(B) $S_{1}=\Pi \backslash \mathfrak{l}$, for some $\mathfrak{l} \subset \Pi$, and we are in Case (b), or

(C) $S_{1}=\cup_{i \in I}\left(\mathfrak{l}_{i} \backslash \mathfrak{q}\right)$, for some $\mathfrak{q} \in \Pi$ and $\mathfrak{l}_{i}$ through $\mathfrak{q}$, and we are in Case (c), or

(D) $k=\mathbb{Q}$, and $\Psi$ is induced from a trivial coloring on $\mathbb{P}^{2}(\mathbb{Z} / p)$.

Note that in Case (B), $\psi \equiv 1$ on the affine plane $\Pi \backslash \mathfrak{l}$.

Lemma 21. In case $(\mathrm{C}), \psi$ is constant on an affine plane, or on $\mathfrak{l}_{i} \backslash \mathfrak{q}$, for all $i \in I$.

Proof. Consider $x \in \Pi \backslash\left(S_{1} \cup \mathfrak{q}\right)$ and lines $\mathfrak{l}$ containing $x$ but not $\mathfrak{q}$. Then $\psi_{\sim} \equiv \psi_{\sim}(x)$ on on $\mathfrak{l} \backslash\left(\mathfrak{l} \cap S_{1}\right)$. Since $S_{1}$ is not an affine plane, there is an $x^{\prime} \in \Pi \backslash\left(S_{1} \cup \mathfrak{l}(x, \mathfrak{q})\right)$. We have $\psi_{\sim}(x)=\psi_{\sim}\left(x^{\prime}\right)$. The union of lines $\mathfrak{l} \subset \Pi, \mathfrak{q} \notin \mathfrak{l}$, through $x, x^{\prime}$, is equal to $\Pi \backslash \mathfrak{q}$. Thus $\psi_{\sim}$ takes only three values $\{1, \psi(x), \psi(\mathfrak{q})\}$ and is constant on $\Pi \backslash\left(S_{1} \cup \mathfrak{q}\right)$. Lemma 20, applied to $\mathfrak{l}$ through $\mathfrak{q}$, implies that $\psi$ is constant on $\mathfrak{l} \backslash \mathfrak{q}$.

We are left with Case (D), when $\Psi$ is induced via some

$$
\rho: \Pi=\mathbb{P}^{2}(\mathbb{Q}) \rightarrow \mathbb{P}^{2}(\mathbb{Z} / p)
$$

from a trivial coloring

$$
\mathfrak{c}: \mathbb{P}^{2}(\mathbb{Z} / p) \rightarrow\{1,2,3\}
$$

in the sense of Proposition 4. Put

$$
\bar{S}_{i}=\mathfrak{c}^{-1}(i), \quad i=1,2,3 .
$$

Note that $S_{1}$ is a finite union of subsets $\mathbb{Z}_{(p)}+\mathbb{Z}_{(p)}$ and does not contain a complete line $\mathfrak{l}$. Consider shifts $\Pi_{z}:=z^{-1} \cdot \Pi$, for $z \in \Pi$. 
Lemma 22. For every $z \in \Pi$, the restriction of $\psi_{\sim}$ to $\Pi_{z}$ is induced from $\mathbb{P}^{2}(\mathbb{Z} / p)$.

Proof. We subdivide (D) into subcases:

(D1) For every $z$ and every splitting $\Pi_{z}=S_{1} \sqcup S_{2} \sqcup S_{3}$, where $S_{2}, S_{3}$ are unions of algebraic dependency classes, the set $\bar{S}_{1} \subset \mathbb{P}^{2}(\mathbb{Z} / p)$ is either a point, an affine line, or an affine plane.

(D2) Otherwise: for some $\Pi_{z}$ this is not the case.

First we treat (D1). Fix $\Pi=\Pi_{z}$ and a decomposition $\Pi=S_{1} \sqcup S_{2} \sqcup S_{3}$; we have

$$
\bar{\Psi}: \mathbb{P}^{2}(\mathbb{Z} / p) \rightarrow\{1,2,3\}
$$

and

$$
\mathbb{P}^{2}(\mathbb{Z} / p)=\sqcup_{i=1}^{3} \bar{S}_{i}, \quad S_{i}=\rho^{-1}\left(\bar{S}_{i}\right) \subset \Pi .
$$

By assumption (D1), we have 3 cases.

- $\bar{S}_{1}=\overline{\mathfrak{q}}$, for some $\overline{\mathfrak{q}} \in \mathbb{P}^{2}(\mathbb{Z} / p)$. For $x \in \Pi \backslash S_{1}$ and $\mathfrak{l}=\mathfrak{l}(\mathfrak{q}, x)$, with $\rho(\mathfrak{q})=\overline{\mathfrak{q}}, \psi_{\sim}$ is constant on $\mathfrak{l} \backslash\left(\mathfrak{l} \cap S_{1}\right)$, by (TC). Apply this to all $\mathfrak{l}\left(\mathfrak{q}, x_{1}\right)$, where $x_{1}$ runs over $S_{1}$, to conclude that $\psi_{\sim}$ is constant on preimages of affine lines $(\overline{\mathfrak{l}} \backslash \overline{\mathfrak{q}})$, with $\overline{\mathfrak{q}} \in \overline{\mathfrak{l}}$, hence is induced from $\mathbb{P}^{2}(\mathbb{Z} / p)$.

- $\bar{S}_{1}=\overline{\mathfrak{l}} \backslash \overline{\mathfrak{q}}$, for some $\overline{\mathfrak{l}} \subset \mathbb{P}^{2}(\mathbb{Z} / p)$ and $\overline{\mathfrak{q}} \in \overline{\mathfrak{l}}$. Then $\bar{S}_{1}, \bar{S}_{2}$ and $\bar{S}_{3}$ form a flag on $\mathbb{P}^{2}(\mathbb{Z} / p)$ : all points projecting to $\mathbb{P}^{2}(\mathbb{Z} / p) \backslash \overline{\mathfrak{l}}$ belong to the same algebraic dependency class because each pair of such points can be connected by a pair of lines which intersect $S_{1}$. Lemma 15 reduces the proof to the previous case, after changing to a different $\psi_{\sim}$-compatible lattice.

- $\bar{S}_{1}=\mathbb{P}^{2}(\mathbb{Z} / p) \backslash \overline{\mathfrak{l}}$, for some line $\overline{\mathfrak{l}} \in \mathbb{P}^{2}(\mathbb{Z} / p)$. This reduces to the case $\bar{S}_{1}=\overline{\mathfrak{q}}$.

We pass to (D2) and fix a plane $\Pi=\Pi_{z}$, with a splitting

$$
\Pi=S_{1} \sqcup S_{2} \sqcup S_{3},
$$

violating (D1). Then there exist a point $\overline{\mathfrak{q}} \in \mathbb{P}^{2}(\mathbb{Z} / p)$ and a set $\left\{\overline{\mathfrak{l}}_{i}\right\}_{i \in I}$ of at least two lines passing through $\overline{\mathfrak{q}}$ such that $\bar{S}_{1}=\cup_{i \in I}\left(\overline{\mathfrak{l}}_{i} \backslash \overline{\mathfrak{q}}\right)$. Moreover, we may assume that $\bar{S}_{2}=\overline{\mathfrak{q}}$, then $\bar{S}_{3}$ has the same structure as $\bar{S}_{1}$, i.e., a union of affine lines containing $\overline{\mathfrak{q}}$ in their closure.

We claim that $\psi_{\sim}$ is constant on $S_{3}$ : consider $\overline{\mathfrak{q}}_{3}, \overline{\mathfrak{q}}_{3}^{\prime} \in \bar{S}_{3}$ not lying on a line through $\overline{\mathfrak{q}}$. Let $\mathfrak{q}_{3}, \mathfrak{q}_{3}^{\prime}$ be any points projecting to $\overline{\mathfrak{q}}_{3}, \overline{\mathfrak{q}}_{3}^{\prime}$. Since $\overline{\mathfrak{l}}\left(\overline{\mathfrak{q}}_{3}, \overline{\mathfrak{q}}_{3}^{\prime}\right) \cap \bar{S}_{1} \neq \emptyset$, the line $\mathfrak{l}\left(\mathfrak{q}_{3}, \overline{\mathfrak{q}}_{3}^{\prime}\right)$ intersects $S_{1}$, thus $\mathfrak{q}_{3} \sim \mathfrak{q}_{3}^{\prime}$. By assumption on $\bar{S}_{3}$, any two points in $S_{3}$ can be connected by a chain of such lines.

Note that $\psi_{\sim}$ is constant on $S_{2}$ : consider

$$
\mathfrak{q}_{1}, \mathfrak{q}_{2} \quad \text { with } \quad \rho\left(\mathfrak{q}_{1}\right)=\rho\left(\mathfrak{q}_{2}\right)=\overline{\mathfrak{q}} \in \bar{S}_{2} .
$$


Then $\psi\left(\mathfrak{q}_{1}\right)=\psi\left(\mathfrak{q}_{2}\right)$. Indeed, consider $\mathfrak{l}_{5}=\mathfrak{l}\left(\mathfrak{q}_{1}, x_{1}\right)$ and $\mathfrak{l}_{6}=\mathfrak{l}\left(\mathfrak{q}_{2}, x_{2}\right)$, where $\rho\left(x_{i}\right)=\bar{x}_{i} \in \bar{S}_{1}, \bar{x}_{1} \neq \bar{x}_{2}$. Hence $\mathfrak{q}_{3}:=\mathfrak{l}_{5} \cap \mathfrak{l}_{6}$ projects to $\overline{\mathfrak{q}}$. Thus $\psi\left(\mathfrak{q}_{1}\right)=\psi\left(\mathfrak{q}_{3}\right)=\psi_{\sim}\left(\mathfrak{q}_{2}\right)$. Thus $\psi_{\sim}$ is constant on $S_{2}$, hence $\psi_{\sim}$ is induced from $\mathbb{P}^{2}(\mathbb{Z} / p)$.

Lemma 23. The map $\psi_{\Pi}$ is induced from $\bar{\psi}_{\Pi}: \mathbb{P}^{2}(\mathbb{Z} / p) \rightarrow A$ which is of the type (a), (b), or (c).

Proof. By Lemma 22, we have the following possibilities:

(1) $\psi_{\sim}$ is induced from a flag map on $\mathbb{P}^{2}(\mathbb{Z} / p)$ and we can assume that $\bar{S}_{1}=\overline{\mathfrak{q}}$, by Lemma 15 ,

(2) $\psi_{\sim}$ is induced from a map on $\mathbb{P}^{2}(\mathbb{Z} / p)$ which is constant on affine lines $\overline{\mathfrak{l}}_{i} \backslash \overline{\mathfrak{q}}$, with $\overline{\mathfrak{q}} \in \overline{\mathfrak{l}}$, and $\bar{S}_{1}=\overline{\mathfrak{q}}$;

(3) $\psi_{\sim}$ is induced from a map on $\mathbb{P}^{2}(\mathbb{Z} / p)$ which is constant on affine lines $\overline{\mathfrak{l}}_{i} \backslash \overline{\mathfrak{q}}$, with $\overline{\mathfrak{q}} \in \overline{\mathfrak{l}}$, and $\bar{S}_{1}$ contains $\overline{\mathfrak{l}}_{i} \backslash \overline{\mathfrak{q}}, i=1,2$.

Case (1): We may assume that $\bar{S}_{3}=\mathbb{P}^{2}(\mathbb{Z} / p) \backslash \overline{\mathfrak{l}}$, for some $\overline{\mathfrak{l}}$ with $\overline{\mathfrak{q}} \in \overline{\mathfrak{l}}$, and $\overline{\mathfrak{l}} \backslash \overline{\mathfrak{q}}=\bar{S}_{2}$. Let $\mathfrak{l}$ be disjoint from $S_{1}$ and $\mathfrak{q}, \mathfrak{q}^{\prime} \in \mathfrak{l} \cap S_{3}$. Since $\psi_{\sim}(\mathfrak{q})=$ $\psi_{\sim}\left(\mathfrak{q}^{\prime}\right)$ and $\mathfrak{l}$ intersects $S_{2}, \psi(\mathfrak{q})=\psi\left(\mathfrak{q}^{\prime}\right)$, by Lemma 19. Since any two points in $S_{3}$ can be connected by a chain of lines disjoint from $S_{1}, \psi$ is constant on $S_{3}$. It is also constant on $\rho^{-1}\left(\overline{\mathfrak{q}}_{2}\right)$, for $\overline{\mathfrak{q}}_{2} \in \bar{S}_{2}$. Indeed, if $\mathfrak{q}_{2}, \mathfrak{q}_{2}^{\prime}$ are distinct points projecting to $\overline{\mathfrak{q}}_{2}$ and $\mathfrak{l}, \mathfrak{l}^{\prime}$ lines containing $\mathfrak{q}_{2}$, resp. $\mathfrak{q}_{2}^{\prime}$, avoiding $S_{1}$ and projecting to distinct lines in $\mathbb{P}^{2}(\mathbb{Z} / p)$, then $\mathfrak{q}_{2}^{\prime \prime}:=\mathfrak{l} \cap \mathfrak{l}^{\prime}$ also projects to $\overline{\mathfrak{q}}_{2}$. Thus $\psi\left(\mathfrak{q}_{2}\right)=\psi\left(\mathfrak{q}_{2}^{\prime}\right)=\psi\left(\mathfrak{q}_{2}^{\prime \prime}\right)$.

Case (2): $\bar{S}_{1}=\overline{\mathfrak{q}}$. If $\psi$ is induced from a noninjective $\bar{\psi}: \mathbb{P}^{2}(\mathbb{Z} / p) \rightarrow$ $A, \psi$ is constant on the preimage of every affine line $\overline{\mathfrak{l}} \backslash \overline{\mathfrak{q}}$, by the same analysis over a finite field.

If there exist $y_{1}, y_{2}$, projecting to the same points $\bar{x} \in \overline{\mathfrak{l}} \backslash \overline{\mathfrak{q}}$, with $\psi\left(y_{1}\right) \neq \psi\left(y_{2}\right)$, let $z_{1}, z_{2}$ have $\psi_{\sim}\left(z_{i}\right) \neq \psi_{\sim}\left(y_{i}\right)$. Consider

$$
z:=\mathfrak{l}\left(y_{1}, z_{1}\right) \cap \mathfrak{l}\left(y_{2}, z_{2}\right)
$$

so that $\rho(z)=\bar{x}$. Then $\psi\left(y_{1}\right)=\psi(z)=\psi\left(y_{1}\right)$, by Lemma 20. Since all points over $\bar{x}$ are connected by a chain of lines of such type, $\psi$ is constant on $\rho^{-1}(\bar{x})$.

Case (3): The argument of Case (1) shows that $\psi$ is constant on the preimage of any affine line $\overline{\mathfrak{l}} \backslash \overline{\mathfrak{q}}$ contained in $\bar{S}_{3}$. Indeed, let $z_{1}, z_{2} \in S_{3}$ and consider $\mathfrak{l}:=\mathfrak{l}\left(z_{1}, z_{2}\right)$. It intersects $S_{2}$ and hence $\psi\left(z_{1}\right)=\psi\left(z_{2}\right)$. Thus $\psi$ is induced from $\mathbb{P}^{2}(\mathbb{Z} / p) \backslash \mathfrak{q}=\bar{S}_{2}$. Let $\mathfrak{q}, \mathfrak{q}^{\prime}$, projecting $\overline{\mathfrak{q}}$. Consider lines $\mathfrak{l}\left(\mathfrak{q}, z_{1}\right)$ and $\mathfrak{l}\left(q^{\prime}, z_{2}\right)$ with $z_{i} \in S_{3}$, which intersect in $\mathfrak{q}^{\prime \prime}, \rho\left(\mathfrak{q}^{\prime \prime}\right)=\overline{\mathfrak{q}}$. Then $\psi(\mathfrak{q})=\psi\left(\mathfrak{q}^{\prime \prime}\right)=\psi\left(\mathfrak{q}^{\prime}\right)$, by Lemma 20. Since any 
pair of points over $\bar{q}$ can be connected by a chain of such lines, $\psi$ is constant on $\rho^{-1}(\overline{\mathfrak{q}})$.

This concludes the proof of Proposition 18 .

Remark 24. This Lemma is similar to [6] and [5, Lemma 13].

\section{LiNES OF INJECTIVITY}

In our analysis of the restriction $\psi_{\mathfrak{l}}$ of

$$
\psi: \mathbb{P}(K) \rightarrow A=L^{\times} / \tilde{l}^{\times}
$$

to lines $\mathfrak{l}=\mathfrak{l}(1, x) \subset \mathbb{P}(K)$ we distinguish the following possibilities:

- $\psi_{\mathfrak{r}}$ is not induced from a map $\bar{\psi}_{\mathfrak{r}}: \mathbb{P}^{1}(\mathbb{Z} / p) \rightarrow A$ and $\psi_{\mathfrak{r}}$ is:

(I) injective

(N) not injective and nonflag

(F) a nonconstant flag map

- $\psi_{\mathfrak{r}}$ is induced from $\bar{\psi}_{\mathfrak{r}}: \mathbb{P}^{1}(\mathbb{Z} / p) \rightarrow A$ and $\bar{\psi}_{\mathfrak{r}}$ is

(I) injective

$(\overline{\mathrm{N}})$ not injective and nonflag

$(\overline{\mathrm{F}})$ a nonconstant flag map

- (C) $\psi_{\mathfrak{r}}$ is constant

Definition 25. Let $\mathfrak{u} \subset \mathbb{P}(K)$ be the union of all lines through 1 , on which $\psi$ is injective and put

$$
\mathfrak{U}:=\{x y \mid x, y \in \mathfrak{u}\} \subseteq \mathbb{P}(K) .
$$

Lemma 26. If $\psi(\mathfrak{u})$ contains at least two algebraically independent elements, then $\mathfrak{U}$ is a group.

Proof. Clearly, $\mathfrak{u}$ and $\mathfrak{U}$ contain $1 \in K^{\times} / k^{\times}$. If $x \in \mathfrak{U}$ then $x^{-1} \in \mathfrak{U}$, by injectivity of $\psi$ on $\mathfrak{l}\left(1, x^{-1}\right)$. Furthermore,

$$
x y^{-1} \in \mathfrak{u} \text {, for all } x, y \in \mathfrak{u} \text { such that } \psi(x) \neq \psi(y) .
$$

Indeed, if $\psi(x) \not \psi(y)$ then $\psi$ is injective on $\Pi(1, x, y)$, by Proposition [18, and in particular on $\mathfrak{l}(x, y)=y \cdot \mathfrak{l}\left(1, x y^{-1}\right)$; thus, $x y^{-1} \in \mathfrak{u}$.

If $\psi(x) \sim \psi(y)$, but are not equal in $A$, take $z \in \mathfrak{u}$ such that $\psi(x) \not$ $\psi(z)$. Then $x / z, y / z \in \mathfrak{u}$, as above. Since $\psi(x / z) \not \psi(y / z)$, the same argument shows that $(x / z) /(y / z)=x y^{-1} \in \mathfrak{u}$, proving (13).

To show that $\mathfrak{U}$ is multiplicatively closed it suffices to check that for every $x_{1}, x_{2}, x_{3} \in \mathfrak{u} \backslash\{1\}$ there exist $s_{1}, s_{2} \in \mathfrak{u}$ with $x_{1} x_{2} x_{3}=s_{1} s_{2}$. Note that $\psi\left(x_{i} x_{j}\right) \neq 1$ for some $1 \leq i<j \leq 3$. (Otherwise,

$$
\psi\left(x_{1} x_{2}\right)=\psi\left(x_{1} x_{3}\right)=\psi\left(x_{2} x_{3}\right)=1,
$$


and therefore,

$$
\psi\left(x_{1}^{2}\right)=\psi\left(\left(x_{1} x_{2}\right)\left(x_{1} x_{3}\right) /\left(x_{2} x_{3}\right)\right)=1,
$$

so $\psi\left(x_{1}\right)=1$.) Then, by (13),$x_{i} x_{j} \in \mathfrak{u}$, so we can take $s_{1}:=x_{i} x_{j}$ and $s_{2}:=x_{t}$, where $\{i, j, t\}=\{1,2,3\}$.

Definition 27. Let $\overline{\mathfrak{u}} \subset \mathbb{P}(K)$ be the union of all lines $\mathfrak{l}$ through 1 , such that the restriction of $\psi$ to $\mathfrak{l}$ is induced via an injective map

$$
\bar{\psi}_{\mathfrak{l}}: \mathbb{P}^{1}(\mathbb{Z} / p) \rightarrow A
$$

and put

$$
\overline{\mathfrak{U}}:=\{x y \mid x, y \in \overline{\mathfrak{u}}\} \subseteq \mathbb{P}(K) .
$$

Lemma 28. If $\psi(\overline{\mathfrak{u}})$ contains at least two algebraically independent elements, then $\overline{\mathfrak{U}}$ is group.

Proof. The proof follows the same steps as the proof of Lemma 26.

Lemma 29. Assume $\mathbb{P}(K)$ contains lines of type (I) and one of the types

$$
(\mathrm{N}),(\overline{\mathrm{I}}),(\overline{\mathrm{N}}), \quad \text { or } \quad(\overline{\mathrm{F}}) \text {. }
$$

Then there exists a one-dimensional subfield $E \subset L$ such that for all lines $\mathfrak{l} \subset \mathbb{P}(K)$ of type $(\mathrm{I}),(\mathrm{N}),(\overline{\mathrm{I}}),(\overline{\mathrm{N}})$, or $(\overline{\mathrm{F}})$ we have

$$
\psi(\mathfrak{l}) \subset E^{\times} / \tilde{l}^{\times} .
$$

In particular, if $\psi(\mathfrak{u})$ contains algebraically independent elements, lines of type $(\mathrm{N}),(\overline{\mathrm{I}}),(\overline{\mathrm{N}})$, and $(\overline{\mathrm{F}})$ do not exist.

Proof. Let $\mathfrak{l}=\mathfrak{l}(1, y)$ be a line of type (I).

If there exists another line $\mathfrak{l}\left(1, y^{\prime}\right)$ of type (I) with $\psi(y) \nsim \psi\left(y^{\prime}\right)$, i.e., $\psi(\mathfrak{u})$ contains independent elements, then lines of the listed type cannot exist, indeed, if $\mathfrak{l}(1, x)$ is of types listed in (14), we apply Proposition 18 to $\Pi=\Pi(1, x, y)$. In Case (a), the exceptional line is $\mathfrak{l}(1, y)$ and hence the restriction of $\psi$ to any other line is either constant or of type $(\mathrm{F})$, contradiction. In Case (b), all lines are either of type (I) or (F), again a contradiction. Case (c) does not apply, since $\mathfrak{l}(1, y)$ is not induced from a map $\mathbb{P}^{2}(\mathbb{Z} / p) \rightarrow A$. Contradiction.

If $\psi(\mathfrak{u})$ does not contain algebraically independent elements, but one of the lines $\mathfrak{l}(1, x)$ in (14) is such that $\psi(x) \nsim \psi(y)$ then we apply the same argument to $\Pi(1, x, y)$ and obtain the same contradiction.

Lemma 30. Assume that $\psi(\mathfrak{u})$ contains algebraically independent elements. Consider $\mathfrak{l}:=\mathfrak{l}(1, y) \nsubseteq \mathbb{\mathfrak { u }}$ and assume that $\mathfrak{l} \cap \mathfrak{U}$ consists of at least two points $1, z^{\prime}$. Then $\mathfrak{l} \cap \mathfrak{U}$ is either $\mathfrak{l}$ or $\mathfrak{l} \backslash \mathfrak{q}$, for some point $\mathfrak{q} \in \mathfrak{l}$. 
Proof. Assume that $\psi_{\mathfrak{r}}$ is not constant, e.g., $\psi(y) \neq 1$. By assumption, there is an $x$ with $\mathfrak{l}(1, x) \subset \mathfrak{u}$ with $\psi(x) \nsim \psi(y)$. We apply Proposition 18 to $\Pi:=\Pi(1, x, y)$. We are not in Case (c) of this lemma. If we are in Case (a), then $\psi$ is constant on $\Pi \backslash \mathfrak{l}(1, x)$, which implies that $\mathfrak{l}$ is of type $(\mathrm{F})$. If we are in Case (b), then the exceptional point $\mathfrak{q}=y$, and $\psi$ is constant, on the complement to $\mathfrak{q}$, on every line through $\mathfrak{q}$, thus $\mathfrak{l}$ is of type $(\mathrm{F})$.

Put $z^{\prime}=t / t^{\prime}$, with $t, t^{\prime} \in \mathfrak{u}$. If $\psi(t) \neq \psi\left(t^{\prime}\right)$ then Equation (13) implies that $z^{\prime} \in \mathfrak{u}$, a contradiction. Thus $\psi_{\mathfrak{l}}$ is either constant or contains one point $y^{\prime} \notin \mathfrak{U}$. In Case $(\mathrm{a}), \psi$ is constant on $\Pi \backslash \mathfrak{l}(1, x)$, thus identically 1 on the line $\mathfrak{l}$. In Case (b), $\psi$ is injective on every line not containing the exceptional point $\mathfrak{q}$, in particular on $\mathfrak{l}\left(1, t^{\prime} / t^{\prime \prime}\right)$, for all $t^{\prime \prime}$, thus $t^{\prime} / t^{\prime \prime} \in \mathfrak{u}$, thus $t^{\prime \prime} \in \mathfrak{U}$. Taking $t^{\prime \prime} \in \mathfrak{l} \backslash \mathfrak{q}$ we obtain the claim.

Now assume that $\psi_{\mathfrak{l}}$ is constant. We claim that $\mathfrak{l} \backslash(\mathfrak{l} \cap \mathfrak{U})$ contains at most one point. Assume otherwise, and pick $w_{1}, w_{2}$ in this set. Note that $\psi$ is injective on every line $\mathfrak{l}\left(u^{\prime}, t^{\prime}\right) \subset \Pi$, with $t^{\prime} \in \Pi(1, x, y) \cap \mathfrak{u}$, $t^{\prime} \neq 1$, and any point $u^{\prime} \in \mathfrak{l} \cap \mathfrak{U}$. Indeed, we can represent $u^{\prime}=w / w^{\prime}$, with $w, w^{\prime} \in \mathfrak{u}$ and with $\psi(w)=\psi\left(w^{\prime}\right) \not \psi\left(t^{\prime}\right)$. Then $t^{\prime} w^{\prime} / w \in \mathfrak{u}$ and $\mathfrak{l}\left(t^{\prime} w^{\prime} / w, 1\right) \subset \mathfrak{u}$. The converse is also true, and $(\Pi \backslash \mathfrak{l}) \subset \mathfrak{u}$. Indeed, consider lines through $u^{\prime}$ which are not equal to $\mathfrak{l} ; \psi$ is injective on such lines.

Now consider two families of lines: those passing through $w$ (except $\mathfrak{l}$ ), and those throgh $w^{\prime}$ (again, except $\mathfrak{l}$ ). All such lines are of type $(\mathrm{F})$, with generic value $\neq 1$, since $\psi$ does not take value 1 on $\Pi \backslash \mathfrak{l}$. Consider lines $\mathfrak{l}(w, v)$ and $\mathfrak{l}\left(w^{\prime}, v\right)$ from these families, with $v \in(\Pi \backslash \mathfrak{l})$. The generic $\psi$-value on these lines is the same and equal $\psi(v)$. A line through $u^{\prime}$, which does not contain $v$ cannot be of type (I), since it intersects lines $\mathfrak{l}(w, v)$ and $\mathfrak{l}\left(w^{\prime}, v\right)$ in distinct points, but taking the same value on these points, contradicting the established fact that such lines are of type (I).

Lemma 31. Assume $\mathbb{P}(K)$ contains lines of type $(\overline{\mathrm{I}})$ and there exist lines of type $(\mathrm{I})$, or $(\mathrm{N})$, or $(\overline{\mathrm{N}})$. Then $\psi(\overline{\mathfrak{u}})$ does not contain algebraically independent elements.

Proof. Assume the contrary. Let $\mathfrak{l}(1, x)$ be a line of type (I) or (N). Then there exists an $y \in \overline{\mathfrak{u}}$ such that $\psi(y) \nsim \psi(x)$. We apply Proposition 18 to $\Pi=\Pi(1, x, y)$ and obtain a contradiction as in the proof of Lemma 29,

Let $\mathfrak{l}(1, x)$ be of type $(\overline{\mathrm{N}})$. We claim that $\Pi$ does not contain lines of type $(\mathrm{F})$. To exclude this possibility, let $\mathfrak{l}=\mathfrak{l}(z, t) \in \Pi$ be such a line with generic $\psi$-value equal to $s \in A$. 
Take points $x_{1}, x_{2} \in \mathfrak{l}(1, x)$ such that $\psi\left(x_{1}\right) \neq \psi\left(x_{2}\right)$, this is possible since $\psi$ takes at least two values on $\mathfrak{l}(1, x)$. Choose $y_{1}, y_{2} \in \mathfrak{l}(1, y)$ and $\psi\left(y_{1}\right) \neq \psi\left(y_{2}\right)$ and are both not equal to $1 \in A$, this is possible because $\psi$ takes at least three values on $\mathfrak{l}(1, y)$ which is of type $(\overline{\mathrm{I}})$.

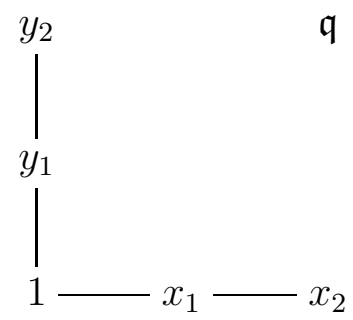

Moreover, we can assume that the lines $\mathfrak{l}_{i j}:=\mathfrak{l}\left(x_{i}, y_{j}\right)$ do not pass through the distinguished point $\mathfrak{q} \in \mathfrak{l}(z, t)$ (where $\psi$ takes the nongeneric value). Thus $\mathfrak{l}_{i j}:=\mathfrak{l}_{i j} \cap \mathfrak{l}(z, t)$ is a generic point of $\mathfrak{l}(z, t)$, which differs from $x_{1}, x_{2}, y_{1}, y_{2}$. Then

$$
\frac{\psi\left(x_{i}\right)}{s} \sim \frac{\psi\left(y_{1}\right)}{s} \sim \frac{\psi\left(y_{2}\right)}{s},
$$

for both $i=1,2$. Hence

$$
1 \neq \frac{\psi\left(x_{1}\right)}{\psi\left(x_{2}\right)} \sim \frac{\psi\left(y_{1}\right)}{\psi\left(y_{2}\right)} \neq 1
$$

Therefore, $\psi(x) \sim \psi(y)$, contradiction.

Thus, for every $\mathfrak{l} \subset \Pi(1, x, y)$ the restriction $\psi_{\mathfrak{l}}$ is induced from a map $\bar{\psi}_{\mathfrak{l}}: \mathbb{P}^{1}(\mathbb{Z} / p) \rightarrow A$. Now we apply Lemma 23. In Case (a) of that Lemma, the exceptional line is $\mathfrak{l}(1, y)$ and of type $(\overline{\mathrm{I}})$ and hence the restriction of $\psi$ to any other line is either constant or of type $(\overline{\mathrm{F}})$, contradiction the assumption that $\mathfrak{l}(1, x)$ is of type $(\overline{\mathrm{N}})$. Cases $(\mathrm{b})$ and (c) are excluded: $\psi$ is not induced from an injective map, nor a flag map on $\mathfrak{l}(1, x)$.

Lemma 32. Assume that the pair of lines $(\mathfrak{l}(1, x), \mathfrak{l}(1, y))$ is of one of the following types

$$
(\mathrm{N}, \mathrm{N}), \quad(\mathrm{N}, \overline{\mathrm{N}}),(\mathrm{N}, \overline{\mathrm{F}}), \quad(\overline{\mathrm{N}}, \overline{\mathrm{N}}) .
$$

Then $\psi(x) \sim \psi(y)$.

Proof. Follows from the same arguments as in Lemma 31 and Lemma 29.

Lemma 33. Assume that $\psi(\overline{\mathfrak{u}})$ contains algebraically independent elements. Consider $\mathfrak{l}:=\mathfrak{l}(1, z) \nsubseteq \overline{\mathfrak{u}}$, and assume that $\mathfrak{l} \cap \overline{\mathfrak{U}}$ consists of at least two points $1, z^{\prime}$. Then $\psi\left(z^{\prime}\right)=1$ and $\mathfrak{l} \cap \overline{\mathfrak{U}}$ is either

(1) $\mathfrak{l}$; 
(2) an affine line, with $\psi$ not constant on $\mathfrak{l}$;

(3) projectively equivalent to $\mathbb{Z}_{(p)} \subset \mathbb{P}^{1}(\mathbb{Q})$;

(4) an affine line and $\psi$ is constant on $\mathfrak{l}$.

Proof. Assume that $\mathfrak{l} \notin(\mathrm{C})$. Write $z^{\prime}=x / x^{\prime}$ with $x, x^{\prime} \in \overline{\mathfrak{u}}$.

- If $\psi(x) \neq \psi\left(x^{\prime}\right)$ then $x / x^{\prime} \in \overline{\mathfrak{u}}$, by Equation [13, thus $\mathfrak{l} \subset \overline{\mathfrak{u}} \subset \overline{\mathfrak{U}}$, contradiction, so that $\psi\left(z^{\prime}\right)=1$.

- If $\psi(x)=\psi\left(x^{\prime}\right) \neq 1$, let $t \in \overline{\mathfrak{u}}$ be such that $\psi(t) \not \psi\left(x^{\prime}\right)$, and is also independent from a nontrivial value on $\mathfrak{l}$. Then $t / x^{\prime} \in \overline{\mathfrak{u}}$ and the restriction of $\psi$ to (a shift of) $\mathfrak{l}\left(t, x / x^{\prime}\right)$ is of type $(\overline{\mathrm{I}})$. In particular, $\mathfrak{l}(1, t), \mathfrak{l}\left(t, x / x^{\prime}\right)$ are also of type $(\overline{\mathrm{I}})$, by the same argument as in the proof of Lemma 31.

This lemma implies that $\mathfrak{l}$ is of type $(\mathrm{F})$ or $(\overline{\mathrm{F}})$.

- $\mathfrak{l} \in(\mathrm{F})$. In the notation of Proposition 18, $\psi$ is of type b) on $\Pi(1, z, t)$ and the restriction of $\psi$ to every line in $\Pi(1, z, t)$, not passing through a distinguished point $\mathfrak{q} \in \mathfrak{l}$, with $\psi(\mathfrak{q}) \neq 1$, is of type $(\overline{\mathrm{I}})$, which implies that $\mathfrak{l} \backslash \mathfrak{q} \subset \mathfrak{U}$, i.e., we are in Case $(\mathrm{V})$.

- $\mathfrak{l} \in(\overline{\mathrm{F}})$. In this case, $\Pi(1, t, z)$ does not contain lines of type $(\mathrm{F})$, because otherwise, by Proposition 18, $\mathfrak{l}$ will also be of type $(\mathrm{F})$. Hence $\psi$ is induced from $\mathbb{P}^{1}(\mathbb{Z} / p)$ on any line in $\Pi(1, t, z)$ and there are two independent values of $\psi$ on $\Pi(1, z, t)$ not equal to 1. Then $\psi$ on $\Pi(1, z, t)$ is induced from $\bar{\psi}: \mathbb{P}^{2}(\mathbb{Z} / p) \rightarrow A$, by Lemma 23,

The map $\bar{\psi}$ is injective on $\mathfrak{l}\left(1, t^{\prime}\right)$ and $\mathfrak{l}\left(t^{\prime}, z_{1}^{\prime}\right)$, where both $t^{\prime}, z_{1}^{\prime}$ are the images of $t, z^{\prime}$ under the reduction map, and a flag map on $\mathfrak{l}\left(1, z_{1}\right)$, where $z_{1}$ is the image of $z$ in $\mathbb{P}^{2}(\mathbb{Z} / p)$. Thus $\psi$ is induced from type $\mathrm{b}$ ), and hence $\mathfrak{U} \cap \mathfrak{l}$ consists of $y$, with $\psi(y)=1$, a set projectively equivalent to $\mathbb{Z}_{(p)} \subset \mathbb{P}^{1}(\mathbb{Q})$, and we are in Case $(\mathrm{P})$.

Assume that $\mathfrak{l} \in(\mathrm{C})$. Here the difficulty is that $\psi(\Pi(1, z, t))$ does not contain algebraically independent elements and we cannot apply Lemma 23. Note that $\mathfrak{l}(t, s)$, for $s=r / r^{\prime}, r, r^{\prime} \in \mathfrak{u}, s \in \mathfrak{l}$, are of type $(\overline{\mathrm{I}})$, by the argument above.

Then any line $\mathfrak{l}\left(t^{\prime}, u^{\prime}\right) \subset \Pi(1, z, t)$, with $\psi\left(t^{\prime}\right) \neq \psi\left(u^{\prime}\right)$, is of type $(\overline{\mathrm{I}})$, since $\psi$ takes at least three values on this line. Hence $s:=\mathfrak{l}\left(t^{\prime}, u^{\prime}\right) \cap \mathfrak{l} \in$ $\overline{\mathfrak{U}}$.

On the other hand, if $s^{\prime} \in \mathfrak{l}$ is not in $\overline{\mathfrak{U}}$ then there are at most two values on any line containing $s^{\prime}$, including $\psi\left(s^{\prime}\right)=1$. We split all points into subsets:

(1) $S_{T}:=\{x \mid \psi(x) \neq 1\}$ 
(2) $S_{1}:=\{x \in \overline{\mathfrak{U}} \mid \psi(x)=1\}$;

(3) $S_{2}:=\{x \notin \overline{\mathfrak{U}} \mid \psi(x)=1\}$.

Note that $S_{T}, S_{1} \neq \emptyset$. If $S_{2}=\emptyset$ then $\mathfrak{l} \subset \overline{\mathfrak{U}}$; and $\overline{\mathfrak{U}} \cap \mathfrak{l}$ satisfies the lemma.

Assume that $S_{2} \cap \mathfrak{l} \neq \emptyset$. We claim that every line in $\Pi(1, z, t)$ lies in the union of two of such subsets. Clearly, this holds for $\mathfrak{l}$. Let $\mathfrak{l}^{\prime} \subset \Pi(1, z, t)$ be a different line and put $s:=\mathfrak{l} \cap \mathfrak{l}^{\prime}$. If $s \in \overline{\mathfrak{U}}$, then $\mathfrak{l}(s, t) \subset \overline{\mathfrak{U}}$, by construction, and all points $s \in \mathfrak{l} \cap S_{T}$ are in $\overline{\mathfrak{u}}$ and those with $\psi(s)=1$ in $\overline{\mathfrak{U}}$. In particular, $\mathfrak{l}(s, t) \subset S_{T} \sqcup S_{1}$. If $s^{\prime} \in \mathfrak{l}$ is in $S_{2}$, then $\mathfrak{l}\left(s^{\prime}, x\right)$ is of type $(\mathrm{F}),(\overline{\mathrm{F}})$ or $(\mathrm{C})$, and hence $\psi$ takes at most two values on $\mathfrak{l}\left(s^{\prime}, x\right)$, including $\psi\left(s^{\prime}\right)=1$.

If $s_{2} \in \mathfrak{l}\left(s^{\prime}, x\right), \psi\left(s_{2}\right)=1, x \in \overline{\mathfrak{u}}, \psi(x) \neq 1$, then $s_{2} \in S_{2}$. Otherwise, if $s_{2} \in \mathfrak{U}, x \in \overline{\mathfrak{u}}$, and then $\psi$ is injective on $\mathfrak{l}\left(s^{\prime}, x\right)=\mathfrak{l}\left(s_{2}, x\right)$, by the argument above. Hence $s_{2} \in S_{2}$. Thus $\mathfrak{l}\left(s_{2}, t^{\prime}\right)$, with $t^{\prime} \in \mathfrak{l}(s, t)$, is contained either in $S_{2} \sqcup S_{T}$ or $S_{1} \sqcup S_{2}$.

Any $y \in \Pi(1, z, t)$, with $\psi(y) \neq 1$, is contained in $\overline{\mathfrak{u}}$. Indeed, consider $\mathfrak{l}\left(y, y^{\prime}\right)$, with $\psi(y) \neq \psi\left(y^{\prime}\right), y^{\prime} \in \mathfrak{l}(t, s), \psi\left(y^{\prime}\right) \neq 1$, and $s_{y}:=\mathfrak{l}\left(y, y^{\prime}\right) \cap$ $\mathfrak{l}(1, z)$. Then $\psi\left(s_{y}\right)=1$, hence $y^{\prime} / s_{y} \in \overline{\mathfrak{u}}$, and $\psi$ is injective on $\mathfrak{l}\left(y, y^{\prime}\right)$. Since $y^{\prime} \in \overline{\mathfrak{u}}$, we find that $y \in \overline{\mathfrak{u}}$ and $s_{y} \in S_{1}$.

Thus $S_{T} \subset \overline{\mathfrak{u}}$ and any line $\mathfrak{l}(y, s)$, with $\psi(s)=1$, is either contained in $S_{2} \sqcup S_{T}$ or in $S_{T} \sqcup S_{1}$. This implies that any $\mathfrak{l}\left(s, s_{2}\right)$, with $s \in S_{1}, s_{2} \in S_{2}$, is contained in $S_{1} \sqcup S_{2}$. Note that none of the lines is contained in one of the subsets $S_{T}, S_{1}, S_{2}$. By Proposition 5, the decomposition $\Pi=S_{T} \sqcup S_{1} \sqcup S_{2}$ is either

(1) a cone over the decomposition of $\mathfrak{l}(t, s)$ into the intersection with $S_{T}$ and $S_{1}$, and $S_{2}$ is just one point in $\mathfrak{l}$;

(2) or is induced from a decomposition of $\mathbb{P}^{2}(\mathbb{Z} / p)$ over the residue of $\mathfrak{l}$, with $S_{1}$ equal to the preimage of a point, and hence $S_{2} \cap \mathfrak{l}$ is projectively equivalent to $\mathbb{Z}_{(p)}$.

\section{Proof of the MAIN THEOREM}

We turn to the proof of Theorem 2, describing homomorphisms

$$
\psi: \mathbb{P}(K) \rightarrow \mathbb{P}(L)
$$

preserving algebraic dependence. There are two possibilities:

(V) $\psi$ factors through a valuation,

(P) $\psi$ factors through a subfield,

described in detail in the Introduction. 
We organize our proof as a case by case analysis, based on types of line, introduced at the beginning of Section 6. We consider two sets of cases as follows.

- Generic cases: $\psi(\mathfrak{u})$ (respectively, $\psi(\overline{\mathfrak{u}})$ ), contains nonconstant algebraically independent elements, i.e., there exist $y_{1}, y_{2} \in$ $\psi(\mathfrak{u})$ (respectively, $\psi(\overline{\mathfrak{u}})$ ) such that $y_{1} \not y_{2}$.

- Degenerate cases: these sets do not contain algebraically independent elements.

In our proof we need the following technical assumption:

(AD) $\psi(\overline{\mathfrak{u}})$ does not contain nonconstant algebraically independent elements.

This is satisfied when $K$ has positive characteristic.

Lemma 34. Assume that $\psi(\mathfrak{u})$ contains nonconstant algebraically dependent elements and that $\mathbb{P}(K)$ contains lines of type $(\mathrm{F})$ and possibly also $(\mathrm{C})$. Then there exists a valuation $\nu$ of $K$ such that $\mathfrak{o}_{\nu}^{\times} \subseteq \mathfrak{U}$ and $\psi\left(\left(1+\mathfrak{m}_{\nu}\right)^{\times}\right)=1$.

Proof. By Lemma 26, $\mathfrak{U} \subset K^{\times} / k^{\times}$is a group, the induced quotient map $K^{\times} / k^{\times} \rightarrow K^{\times} / \mathfrak{U}$ is a nontrivial flag map, by the assumption on the existence of lines of type $(\mathrm{F})$ in $\mathbb{P}(K)$. Thus there is a map

$$
\mathfrak{o}_{\mu}^{\times} \rightarrow K^{\times} \rightarrow \Gamma_{\mu},
$$

for some valuation $\mu$, with the property that $K^{\times} \rightarrow K^{\times} / \mathfrak{U}$ is a composition

$$
K^{\times} \rightarrow \Gamma_{\mu} \stackrel{r_{\mu}}{\longrightarrow} K^{\times} / \mathfrak{U}
$$

Let

$$
\Gamma_{\mu}^{+}:=\nu\left(\mathfrak{o}_{\mu} \backslash 0\right) \subset \Gamma_{\mu}
$$

be the subsemigroup of positive elements and put

$$
\operatorname{Ker}\left(r_{\mu}\right)^{+}:=\operatorname{Ker}\left(r_{\mu}\right) \cap \Gamma_{\mu}^{+} .
$$

- Assume that $\operatorname{Ker}\left(r_{\mu}\right)^{+}=0$. Then for any nonconstant

$$
x \in \mathfrak{o}_{\mu}^{\times} /\left(k^{\times} \cap \mathfrak{o}_{\mu}^{\times}\right) \subset \mathfrak{u}, \quad y \in\left(\mathfrak{m}_{\mu} \backslash 0\right) /\left(k^{\times} \cap \mathfrak{o}_{\mu}^{\times}\right),
$$

the restriction of $\psi$ to $\mathfrak{l}(x, y)$ is a flag map with generic value 1 by Proposition 18, Case (c), with $y=\mathfrak{q}$, hence the result holds for $\nu=\mu$.

- Assume that $\operatorname{Ker}\left(r_{\mu}\right)^{+} \neq 0$. Assume in addition that there exists a $\gamma^{+} \in\left(\Gamma_{\mu}^{+} \backslash \operatorname{Ker}\left(r_{\mu}\right)^{+}\right)$and such that $\gamma^{+}<\gamma^{\prime}$ for some $\gamma^{\prime} \in \operatorname{Ker}\left(r_{\mu}\right)^{+}$. Consider $x \in(\mathfrak{u} \backslash 1)$, with $\mu(x)=\gamma^{\prime}$, and $y \in \mathfrak{o}_{\mu}^{\times} /\left(k^{\times} \cap \mathfrak{o}_{\mu}^{\times}\right)$, with $\mu(y)=\gamma^{+}$. The restriction of $\psi$ to $\mathfrak{l}(1, y) \subset \mathbb{P}(1, x, y)$ is a flag map with generic value 1 . On the 
one hand, $\mathfrak{l}:=\mathfrak{l}(x, y) \not \subset \mathfrak{u}$, hence $\psi_{\mathfrak{l}}$ is a flag map, with generic value $\psi(x)$. On the other hand, the generic value of $\psi$ on $\mathfrak{l}(1, y)$ is 1 , hence $\psi(x+y)=\psi(x)$ and $x+y \in \mathfrak{u}$. We have $\mu(y)<\mu(x)$ and, on $\mathfrak{l}(x, y)$, we have $\mu(x+y)=\mu(y)$, hence $\psi(x+y)=\psi(y)$, contradiction.

This implies that elements of $\operatorname{Ker}\left(r_{\mu}\right)^{+}$are smaller than all elements in $\left(\Gamma_{\mu}^{+} \backslash \operatorname{Ker}\left(r_{\mu}\right)^{+}\right)$. Thus the subgroup of $\Gamma_{\mu}$ generated by $\operatorname{Ker}\left(r_{\mu}\right)^{+}$is an ordered subgroup. The homomorphism $\Gamma_{\mu} \rightarrow$ $\Gamma_{\mu} / \operatorname{Ker}\left(r_{\mu}\right)^{+}$identifies $\Gamma_{\mu} / \operatorname{Ker}\left(r_{\mu}\right)^{+}$with a valuation group $\Gamma_{\nu}$ for some valuation $\nu$ of $K$, and $\psi\left(\left(1+\mathfrak{m}_{\nu}\right)^{\times}\right)=1$.

We can also treat all degenerate cases, i.e., $\psi(\mathfrak{u})$ and $\psi(\overline{\mathfrak{u}})$ do not contain nonconstant algebraically independent elements.

Most degenerate case: no $(\mathrm{I}),(\overline{\mathrm{I}}),(\mathrm{N})$, and $(\overline{\mathrm{N}})$-lines:

- Then $\psi$ is a flag map on all $\mathfrak{l} \subset \mathbb{P}(K)$, hence a flag map, and there exists a valuation $\nu$ such that $\psi$ factors through $\Gamma_{\nu}$, and we are in Case $(\mathrm{V})$ of the Theorem 2 , since $\psi\left(\mathfrak{o}_{\nu}^{\times}\right)=1$.

Degenerate case: no $(\mathrm{I})$ and $(\overline{\mathrm{I}})$-lines, but $(\mathrm{N})$ or $(\overline{\mathrm{N}})$-lines :

- If there exist $(\mathrm{N})$ or $(\overline{\mathrm{N}})$-lines then, by Lemma 32 , there exists a 1-dimensional subfield $L_{1} \subset L$ such that the images of all such lines are contained in $L_{1}^{\times} / l^{\times}$. Consider the induced projection homomorphism

$$
\psi_{1}: \mathbb{P}(K) \rightarrow L^{\times} / l^{\times} \rightarrow L^{\times} / L_{1}^{\times} .
$$

Note that the restriction of $\psi_{1}$ to any line $\mathfrak{l} \in \mathbb{P}(K)$ is a flag map, and there exist lines on which it is a nontrivial flag map, since the image of $\psi$ contains at least two algebraically independent elements. Thus there is a nontrivial valuation $\mu$ of $K$ such that $\psi_{1}$ factors through the value group $\Gamma_{\mu}$.

Degenerate case: there exist (I)-lines $\mathfrak{l}$ and $\psi(\mathfrak{l}) \subset L_{1}^{\times} / l^{\times}$, for some 1-dimensional field $L_{1} \subset L$.

- Let $L_{2}$ be the algebraic closure of $L_{1}$ in $L$. There may also exist lines $\mathfrak{l} \subset \mathbb{P}(K)$ of type $(\mathrm{N}),(\overline{\mathrm{N}}),(\overline{\mathrm{I}})$, or $(\overline{\mathrm{F}})$, with respect to $\psi$, but $\psi(\mathfrak{l}) \subset L_{2}^{\times} / l^{\times}$for all such $\mathfrak{l}$, by Lemma 29, Again, every $\mathfrak{l} \subset \mathbb{P}(K)$ is either of type $(\mathrm{C})$ or $(\mathrm{F})$, with respect to

$$
\psi_{2}: \mathbb{P}(K) \rightarrow L^{\times} / l^{\times} \rightarrow L^{\times} / L_{2}^{\times},
$$


and there exists a nontrivial valuation $\mu$ of $K$ such that $\psi_{2}$ factors through $\Gamma_{\mu}$.

Degenerate case: there are no $(\mathrm{I})$-lines but there exist $(\overline{\mathrm{I}})$-lines whose images are contained in $L_{1}^{\times} / l^{\times}$, for some 1-dimensional subfield of $L$.

- Let $L_{2}$ be its algebraic closure in $L$. There may exist lines of type $(\mathrm{N}),(\overline{\mathrm{N}})$, or $(\overline{\mathrm{I}})$, but their images are contained in $L_{2}^{\times} / l^{\times}$. Every $\mathfrak{l} \subset \mathbb{P}(K)$ is of type $(\mathrm{C})$, $(\mathrm{F})$, or $(\overline{\mathrm{F}})$, with respect to

$$
\psi_{2}: \mathbb{P}(K) \rightarrow L^{\times} / l^{\times} \rightarrow L^{\times} / L_{2}^{\times},
$$

and there exists a nontrivial valuation $\nu$ of $K$ such that $\psi_{2}$ factors through $\Gamma_{\mu}$.

Thus, in all the degenerate cases the homomorphism

$$
\psi_{\ell}: K^{\times} / k^{\times} \rightarrow L^{\times} / L_{2}^{\times},
$$

is a flag map, thus arises from a nontrivial valuation $\mu$,

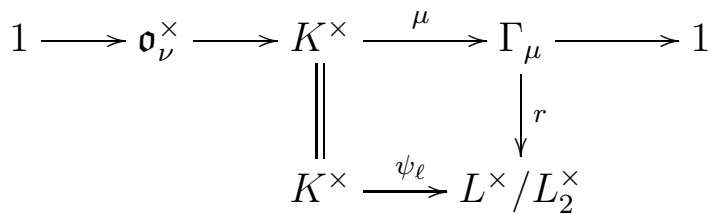

i.e., $\psi_{\ell}=r \circ \mu$ The following Lemmas will show that $\psi$ is either as in (V) or (VP) of Theorem 2 .

Lemma 35. There is a valuation $\nu$ of $K$ and a surjective homomorphism of ordered groups

$$
\Gamma_{\mu} \stackrel{\gamma}{\longrightarrow} \Gamma_{\nu}
$$

such that

(1) $\nu=\gamma \circ \mu: K^{\times} \rightarrow \Gamma_{\nu}$ is a surjective map of ordered groups with $\operatorname{Ker}(\gamma) \subset \operatorname{Ker}(r)$

(2) $\psi\left(\left(1+\mathfrak{m}_{\nu}\right)^{\times}\right)=1$.

Proof. Let $z \in \mathfrak{o}_{\mu}^{\times}$be such that $r(\mu(z)) \neq 0$ and thus $\psi_{\ell}(z) \neq 1 \in$ $L^{\times} / L_{2}^{\times}$. Let $x \in \mathfrak{o}_{\mu}^{\times} \subset \operatorname{Ker}\left(\psi_{\ell}\right)$. We have

$$
\mu(x+a z)=\mu(x), \mu(a) \geq 0,
$$

and $r$ is nonconstant on $\mathfrak{l}(z, x)$. Thus $\psi$ is a flag map on $\mathfrak{l}$, and

$$
\psi(x+a z)=\psi(x)
$$

so that $\psi(1+a z / x)=1$. Note that $z x$ also has $r(\mu(z x)) \neq 0$ and hence we can apply the same to $z x$, obtaining $\psi(1+a z)=1$, for any $z$ with $r(\mu(z))>0$. 
Note that elements $z$ with $\nu(z)=\alpha$ generate additively the subgroup $K_{\alpha} \subset K$. Now the elements of the form $1+z$ with $\nu(1+z)=0$ generate the multiplicative subgroup $\left(1+K_{\alpha}\right)^{\times}$. Indeed, consider

$$
(1+z)\left(1+z^{\prime}\right)=1+z+z^{\prime}+z z^{\prime}=\left(1+z+z^{\prime}\right)\left(1+\frac{z z^{\prime}}{1+z+z^{\prime}}\right),
$$

where $\mu(z)=\mu\left(z^{\prime}\right)$ and $\left(1+z+z^{\prime}\right) \in \mathfrak{o}_{\mu}^{\times}$. Since $\psi_{l}\left(z z^{\prime}\right) \neq 1$ we have

$$
\psi\left(1+\frac{z z^{\prime}}{1+z+z^{\prime}}\right)=1,
$$

by the same argument applied to $z, z^{\prime}$; thus $\psi \equiv 1$ on $\left(1+K_{\alpha}\right)^{\times}$. This implies that $\psi(1+y)=1$, even if $r(\nu(y))=0$ but there is a $z, r(\nu(z)) \neq 1$ and $\nu(z)<\nu(y)$. Consider the subset $\Gamma_{\mu}^{+}, \mu \geq 0$ in $\Gamma_{\mu}$. Since $L^{\times} / L_{2}^{\times}$is torsion-free,

$$
\operatorname{rk}_{\mathbb{Q}}(\operatorname{Ker}(r))<\operatorname{rk}_{\mathbb{Q}}\left(\Gamma_{\mu}\right)
$$

Hence it intersects $\Gamma_{\mu}^{+}$in a proper subsemigroup $\operatorname{Ker}\left(r_{\mu}\right)^{+}$and the subset of elements $s \in \operatorname{Ker}\left(r_{\mu}\right)^{+}$with $s>\mu(x)$ for any $x \in \Gamma_{\mu}^{+}-$ $\operatorname{Ker}\left(r_{\mu}\right)^{+}$.

We are looking a subset of elements $S$ inside $\operatorname{Ker}\left(r_{\mu}\right)^{+}-0$ such that for each $s \in S$ such that $s<u$ for any $u>0$ with $r(\mu(u)) \neq 0$. Note that $S$ has to contain smallest elements in $\Gamma_{\mu}^{+} \backslash 0$ if there are ones. Assume that $s, s^{\prime} \in S, s, s^{\prime}<u, r(u) \neq 0$ and $s+s^{\prime}>u$. Note that $s+s^{\prime}-u>0$ and $s>u-s^{\prime}>0$ but $r\left(u-s^{\prime}\right) \neq 0$ which provides a contradiction. Thus $S$ is an ordered subsemigroup in $\operatorname{Ker}\left(r_{\mu}\right)^{+}-0$ which generates an ordered subgroup $\langle S\rangle$ such that

$$
K^{\times} \rightarrow \Gamma_{\mu} /\langle S\rangle=: \Gamma_{\nu}
$$

is a valuation map for some valuation $\nu$. For this valuation, $\operatorname{Ker}(\nu) \supset$ $\left(1+\mathfrak{m}_{\nu}\right)^{\times}$, by the computation above.

\section{REFERENCES}

[1] F. A. Bogomolov. Abelian subgroups of Galois groups. Izv. Akad. Nauk SSSR Ser. Mat., 55(1):32-67, 1991.

[2] F. A. Bogomolov and Yu. Tschinkel. Commuting elements of Galois groups of function fields. In Motives, polylogarithms and Hodge theory, Part I (Irvine, CA, 1998), volume 3 of Int. Press Lect. Ser., pages 75-120. Int. Press, Somerville, MA, 2002.

[3] F. A. Bogomolov and Yu. Tschinkel. Reconstruction of function fields. Geom. Funct. Anal., 18(2):400-462, 2008.

[4] F. A. Bogomolov and Yu. Tschinkel. Reconstruction of higher-dimensional function fields. Moscow Math. Journal, 11(2):185-204, 2011.

[5] F. A. Bogomolov and Yu. Tschinkel. Galois theory and projective geometry. Comm. Pure Appl. Math., 66(9):1335-1359, 2013. 
[6] A. W. Hales and E. G. Straus. Projective colorings. Pacific J. Math., 99(1):3143, 1982.

[7] F. Pop. On Grothendieck's conjecture of birational anabelian geometry. Ann. of Math. (2), 139(1):145-182, 1994.

Courant Institute of Mathematical Sciences, N.Y.U., 251 Mercer STR., NEW YORK, NY 10012, U.S.A.

National Research University Higher School of Economics, Russian Federation, AG Laboratory, HSE, Usacheva str., Moscow, Russia, 117312

E-mail address: bogomolo@cims.nyu.edu

National Research University Higher School of Economics, Russian Federation, AG Laboratory, HSE, Usacheva str., Moscow, Russia, 117312

E-mail address: marat@mccme.ru

Courant Institute of Mathematical Sciences, N.Y.U., 251 Mercer STR., New York, NY 10012, U.S.A.

Simons Foundation, 160 Fifth Av., New York, NY 10010, U.S.A.

E-mail address: tschinkel@cims.nyu.edu 